\title{
Modeling Bus Bunching and Anti-bunching Control Accounting for Signal Control and Passenger Swapping Behavior
}

\author{
Qi Xin, Rui Fu, and Shaowei Yu \\ Chang'an University \\ Satish V. Ukkusuri \\ Purdue University \\ Rui Jiang \\ Beijing Jiaotong University
}

\begin{abstract}
The conventional bus propagation modeling process has two main shortcomings: bus bunching and extra energy consumption caused by idling at signalized intersections and unexpected speed variation along the route. To overcome these problems simultaneously, an extended bus propagation model and anti-bunching control are proposed in this paper. To extend the time-based bus propagation model, the authors used a finite state machine and an intelligent driver model to establish a spatial-temporal based bus propagation model accounting for dynamic bus motion and passenger swapping behavior between bunched buses. To mitigate bus bunching and improve fuel economy in a connected environment, an anticipated average speed plan was used to improve headway regularity and reduce the chance of encountering a red signal. Then, predictive control accounting for state and control constraints was used to generate a smooth trajectory for connected buses to follow the commands given by anticipated average speed planning. This ensured that connected buses traversed signalized intersections and approached downstream stops efficiently. Simulations show that the proposed model can imitate passenger swapping behavior when bus bunching occurs, and the anti-bunching control can mitigate bus bunching and guide connected buses to traverse signalized intersections and reach downstream stops with less delay.
\end{abstract}

Keywords: Connected bus, bus bunching, signalized intersection, finite state machine, model predictive control, speed planning

(C) 2021 Qi Xin, Rui Fu, Shaowei Yu, Satish V. Ukkusuri, and Rui Jiang https//doi.org/10.5038/2375-0901.23.1.3

ISSN: 1077-291X | Licenced under Creative Commons License Attribution - Noncommercial 4.0

The Journal of Public Transportation is published by the Center for Urban Transportation Research at the University of South Florida 


\section{Introduction}

Many buses are equipped with global positioning systems (GPS) and wireless communication devices to automatically collect and transmit bus position information to the nearest dispatch center. Using these capabilities, bus bunching can be mitigated by executing actions directed by the dispatch center. However, this system does not necessarily reduce idling times at signalized intersections or maintain reliable bus service due to unexpected signal phasing and timing. Today, off-the-shelf wireless communication devices are used as vehicle-to-vehicle (V2V) or vehicle-to-infrastructure (V2I) communication, including dedicated shortrange communication (DSRC), 4G-LTE-V, and 5G. With V2I communications, algorithms can be designed for a connected bus to plan and regulate its speed in advance to make sure it arrives at a signalized intersection during a green phase. As a consequence, speed variations can be reduced upstream and downstream of the intersection, reducing energy consumption. In addition, the connected bus can be more reliable since it can reach the signalized intersection during the green phase by either accelerating or decelerating proactively.

Bus reliability and bus bunching can be described by bus propagation models. In 1964, Newell and Potts proposed a single line bus propagation model that explains the causes of bus bunching. The model assumes a constant ratio between passenger arrival and boarding rate, with a uniform passenger arrival distribution. The en route time is assumed to be constant, indicating the same distance and average speed between two consecutive stops. The conventional bus propagation model includes en route, dwell, and holding times, and a considerable amount of literature considers the impact of passenger behavior on dwell time. Wu, Liu, and Jin (2017) established an analytical bus propagation model accounting for bus overtaking, distributed passenger boarding behavior, and bus capacity. This enhanced model can represent the dynamic passenger queue swapping among bunched buses at stops, but it requires a uniform passenger arrival distribution, which is not always present. Fonzone, Schmöcker, and Liu (2015) proposed a bus propagation model considering reliability-based passenger arrival patterns. This model holds that the passenger arrival rate is related to the anticipated risk-averse waiting time of the passenger at each stop according to non-uniform bus service distribution. Schmöcker et al. (2016) extended the model to a two-lines condition to account for common line and overtaking. This model holds that all passengers will board the front bus if overtaking is forbidden and passengers form balanced queues if overtaking is allowed, indicating that common lines worsen the service regularity if overtaking is forbidden and have positive effects when overtaking is allowed. Dwell time is another important factor attributing to bunching, which should be modeled precisely. Wang et al. (2016) took acceleration and deceleration time, dead time, and time for serving boarding and alighting passengers into account to estimate the dwell time and time lost for serving a stop in China.

To mitigate the bus bunching phenomenon, prediction and control schemes are applied in bus operation. Chien, Ding, and Wei (2002) assumed that passenger waiting time would be greatly improved if passengers could obtain accurate bus arrival information, and then they used two artificial neural networks trained by link-based and stop-based data to predict transit arrival time. Yu et al. (2016) established a least square support vector machine regression based on transit smart card data to detect bus bunching. Wu, Liu, and Jin (2017) used headway holding and schedule holding strategies to alleviate bus bunching when the bus schedule or the anticipated headway is given.

With the development of connected vehicle technology, real-time information about bus operation became available. Based on this, Eberlein, Wilson, and Bernstein (2001) formulated the holding problem as a quadratic program in a rolling horizon scheme, but this scheme is not robust against stochastic demands. To improve the resilience of holding control (HC) and reduce bus bunching, Daganzo and Pilachowski (2011) used an adaptive control system to adjust bus cruising speed in real time just like successive bus pairs connected by springs, 
and the results show that this can generate regular headways as well as faster speed than inserting slacks. Li et al. (2019) combined HC and operating speed control to adjust the bus propagation process and made it robust against congestion delays and passenger demand variations. However, this control strategy assumes that the states of all buses at previous bus stops are known, which is difficult to realize in practice. Headway variations at stops can be treated by optimizing bus stop locations and dispatching headways to improve cost and service reliability (Zhao et al. 2018). By using automatic vehicle location (AVL) schedule adherence data, Cevallos et al. (2011) maximized the density area of the on-time performance range. To minimize bus schedule delays and additional car delays, Yang, Menendez, and Guler (2018) proposed a transit signal priority algorithm using connected vehicle information for multimodal traffic control. To balance delay and equity, Aziz and Ukkusuri (2016) proposed a V2I based signal controller, which can be used to improve the headway regularity of connected bus service without sacrificing equity.

An unexpected signal phase may add extra delays and energy consumption to a human-driven bus, which may in turn result in bus bunching and higher operational cost. Reasons include: (1) the bus is unable to make full use of signal priority, since the controller does not consider dwell time or demand variation; (2) the probability of the bus encountering a red signal is as large as the ratio of red time to cycle length, due to a lack of speed advisory; and (3) unexpected signal phase and service time increase the bus propagation uncertainties, which may result in severe bus bunching. One way to address these problems is to adjust signal timing plans, and the other is to employ a speed advisory system to alleviate bus bunching. Chow and Li (2017) presented a set of signal-based strategies that regulate bus headway by adjusting signal timing plans and the results illustrate that regulating headway errors by signal control can improve bus service reliability. However, signal timing is usually pre-set and does not always change with time or traffic demand. Therefore, some scholars have turned to the speed advisory system to improve bus service regularity.

For the sake of improving mobility and the energy efficiency of a single connected vehicle (CV), research has focused on how to guide CVs to reach an intersection during a green signal. Asadi and Vahidi (2011) established a reference speed planning model based on a map of traffic signals over space and time, and then used model predictive control (MPC) to allow a CV to cruise at the reference speed in order to reduce its probability of encountering a red signal. Li et al. (2015) formulated a Bolza-type optimal control problem (OCP) to minimize fuel consumption. Then they used the Legendre pseudo-spectral method to convert the OCP into a multistage interconnected nonlinear programming problem to determine the optimal engine torque and transmission gear position. To improve the energy economy performance of a partially automated vehicle, Altan et al. (2017) developed an eco-approach and departure system that can receive vehicle-to-everything (V2X) messages from the intersection infrastructure and use piecewise trigonometric-linear functions to automatically generate recommended speed profiles. Aiming at minimizing energy consumption, Han et al. (2018) designed a safetyoriented speed advisory system to derive a real-time analytical speed profile accounting for vehicular distance and speed limit constraints.

Reducing energy consumption should also consider traffic state information and lane changing decisions. Huang et al. (2018) combined traffic state prediction, eco-driving speed planning, and power train control to implement an ecological driving system, which is robust against different traffic scenarios. Zhang et al. (2018) presented an eco-driving control algorithm aimed at minimizing fuel consumption via traffic timing prediction. This study used a Laguerre function based MPC to derive the control input to keep the vehicle moving at its target speed. To consider the queue at the intersection, Xin et al. (2018) established a simple eco-driving model based on the intelligent driver model (IDM), intersection passing decision, and leading vehicle speed recommendation, and found that taking the speed profile generated by the eco-driving model as a speed advisory can reduce idling time and fuel consumption on arterials. To develop a consensus, optimal and safe 
speed advisory to allow a CV to arrive at a signalized intersection during a green phase, Yu et al. (2019) used a consensus-based platoon algorithm to allow platoon followers to obtain similar tracking performances as the platoon leader. Then they used trigonometric and logistic curves to guide the CV platoon leader's smooth approach and departure from the signalized intersection, which can conserve fuel consumption and promote intersection throughput.

To date, the bus propagation model, bus bunching phenomenon, and green phase arrival speed advisory systems have been investigated. However, the bus dynamic motion on the route has not been considered in the bus propagation model. In addition, signalized intersections and bus stops have not been considered simultaneously in the design of most speed advisory algorithms. To overcome these gaps, this paper proposes an extended bus propagation model and an anti-bunching control accounting for signal control and passenger swapping behavior. The main contributions are:

1. A spatial-temporal based bus propagation model is established considering signal control impacts on bus dynamic motion and passenger swapping behavior between bunching buses via a finite state machine.

2. Compared with $\mathrm{HC}$, the proposed speed planning can ensure a connected bus arrives at the signalized intersection during the green phase and reaches the next bus stop more reliably, or with fewer delays.

3. An adaptive time schedule is applied in the anticipated average speed planning (AASP) algorithm, which is more robust on bus bunching alleviation than a constant time schedule.

4. Model predictive control is used to adjust connected bus speed and ensure the bus smoothly follows the commands given by the AASP algorithm.

The remaining sections of the paper are organized as follows. First, the research problem is given, followed by a human-driven bus propagation model that considers bus dynamic motion and passenger swapping behavior. The next section gives the AASP algorithm and MPC based control profile. Numerical simulations are then carried out and some performance metrics are discussed, followed by conclusions.

\section{Problem Statement}

Aiming at improving bus energy efficiency and service reliability, this paper establishes an extended bus propagation model and employs MPC based speed planning to smooth bus trajectory along the bus route. Without loss of generality, some assumptions are made:

1. This paper focuses on one bus line and considers two kinds of buses: a human-driven bus and a connected bus.

2. The human-driven bus is fully controlled by the driver's behavior, thus its motion can be simulated by car following models, such as IDM.

3. The connected bus can receive surrounding vehicle positions, speed, and the downstream signal controller phasing and timing information via V2X communication. This information can be used to design a smooth trajectory to guide the connected bus and regulate its speed in advance, making it arrive at the next signalized intersection during the green phase.

4. The human-driven bus and connected bus drive in a dedicated bus lane, thus speed will not be affected by traffic conditions.

5. Each bus stop is located on the far side of the nearby signalized intersection. When the bus traverses an intersection, there is a shorter distance to the bus stop than to the next intersection. 
6. The passenger arrival rates follow a uniform distribution.

7. Passenger swapping is done automatically among bunching buses.

8. Buses with a front door for boarding and rear door for alighting are considered; hence the dwell time can be calculated as the maximum value of the passenger boarding and alighting times.

9. The alighting demand is assumed to be proportional to the number of onboard passengers. The boarding and alighting rates are assumed to be constant.

10. Neither bus skipping nor overtaking is allowed.

REMARK 1. Bus skipping and overtaking are effective ways to mitigate severe bus bunching. However, the IDM was used in this paper to describe bus following behavior, traversing signalized intersections, and approaching stop behavior, and an iterative method was used to implement passenger swapping, both of which are only valid on a case without skipping or overtaking. Due to the benefit and complexity, the bus propagation model and anti-bunching control accounting for skipping or overtaking are left for future work.

The intelligent driver model is given by equations (1)-(3) to imitate the dynamic motion of the human-driven bus:

$$
\begin{aligned}
& \left\{\begin{array}{l}
\dot{p}_{h}=\min \left(\max \left(v_{h}, 0\right), v_{\max }\right) \\
\dot{v}_{h}=\min \left(\max \left(a\left(1-\left(v_{h} / v_{d}\right)^{\delta}-\left(s_{h d} / s_{h}\right)^{2}\right),-b\right), a\right)
\end{array}\right. \\
& s_{h}=p_{p}-p_{h}-L, \Delta v=v_{h}-v_{p} \\
& s_{h d}=s_{\min }+\max \left(v_{h} T_{s}+\frac{v_{h} \Delta v}{2 \sqrt{a b}}\right)
\end{aligned}
$$

where $p_{h}$ and $v_{h}$ are the host bus location and speed, respectively; $p_{p}$ and $v_{p}$ are the predecessor location and speed, respectively; $s_{h d}$ is the desired distance gap between the host bus front bumper and its predecessor's rear bumper; $L$ is the bus length; $S_{\min }$ is the standstill distance gap; $a$ is the maximum acceptable acceleration limit and $b$ is the comfortable deceleration limit; $\delta$ is the acceleration exponent; $v_{d}$ is the desired speed, which can be determined by big data analysis of bus GPS data; and the typical values of $a, b$, and $\delta$ are given by Kesting et al. (2008).

\section{Human-Driven Bus Propagation Model}

The human-driven bus propagation model is proposed based on a finite state machine. Two types of finite state machines are set, one for the first bus and the other for the followers. The bus propagation model starts from state 0 and then transfers state and performs state action according to the current state and input. Before executing the finite state machines, initial position and speed values are set, as well as the number of onboard passengers and leftover passengers at each bus terminal, initial states for each bus at the terminal are set as state 0 , and initial states for each bus at other bus stops are set as state 4.

\section{Bus Propagation Model for the First Bus}

The bus propagation model for bus 1 includes a five-state machine and a variable updating process. 


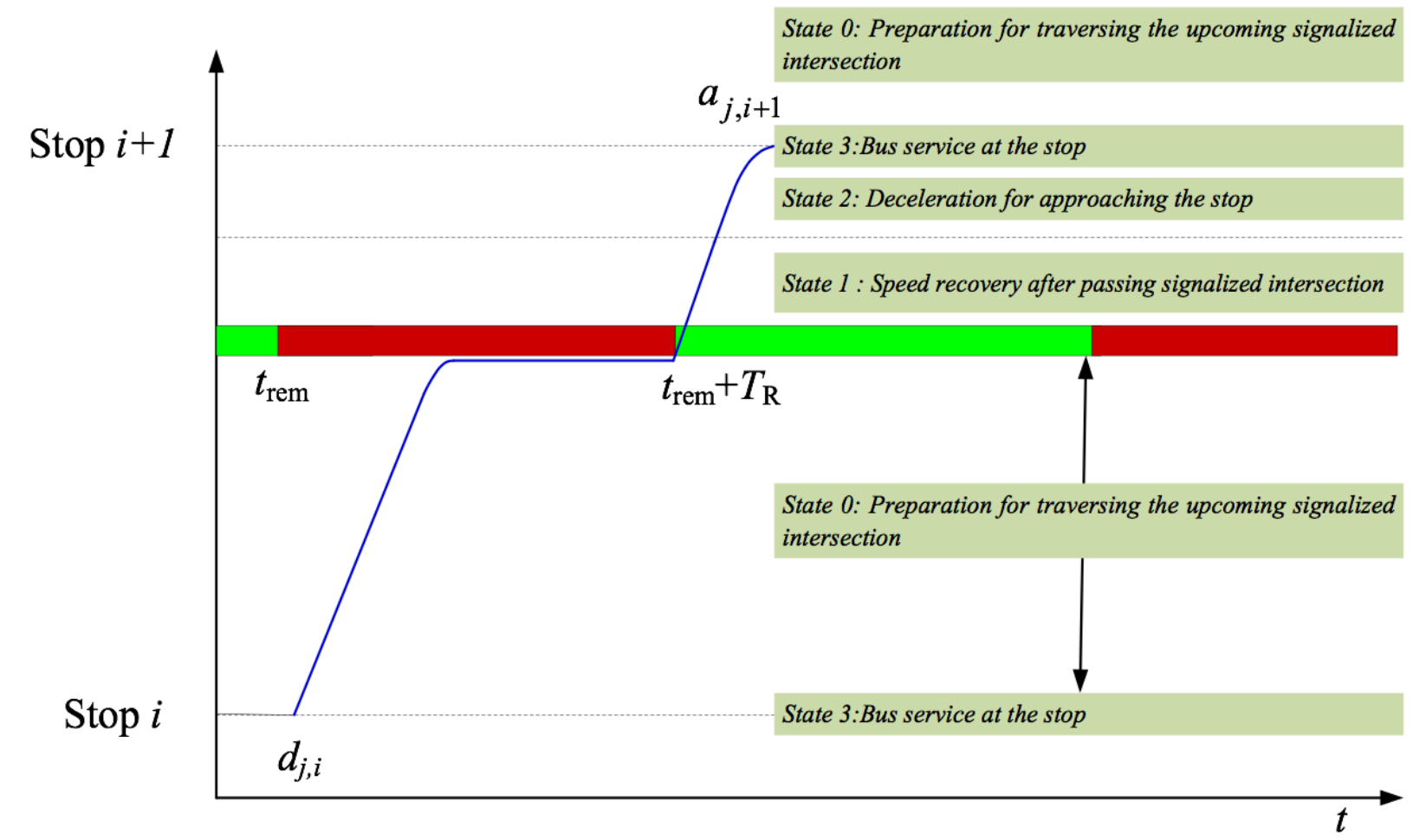

FIGURE 1.

Location based finite state machine model for human-driven bus

\section{Five-State Machine for the First Bus}

As is shown in Figure 1, the five states depend on the location of bus 1, where state 3 means that bus 1 dwells at a bus stop, state 0 means that bus 1 departs the current stop and proceeds to the next intersection, state 1 means that bus 1 departs the signalized intersection and approaches the midpoint between the signalized intersection and next stop, and state 2 means that bus 1 departs the midpoint and runs to the bus stop. State 4 is a spare state used to initiate and activate the finite state machine. The concrete state flow is given as follows.

\section{State 0: Preparation for traversing the next signalized intersection}

State action: If bus 1 is predicted to arrive at signalized intersection $i$ during the green phase, then we set $s_{1, i}=D_{d}, \Delta v_{1, i}=v_{1}-v_{d}$, with $D_{d}$ as the onboard radar maximum detection range. If bus 1 is predicted to arrive at signalized intersection $i$ during the red phase, then we set $s_{1, i}=p_{s, i}-p_{1}, \Delta v_{1, i}=v_{1}$ where $p_{s, i}$ is the location of the stop bar at signalized intersection $i$. If bus 1 is predicted to arrive at signalized intersection $i$ during the yellow phase and is able to stop upstream of the stop bar, meaning that bus 1 satisfies condition (4), then we set $s_{1, i}=p_{s, i}-p_{1}$ and $\Delta v_{1, i}=v_{1}$. Otherwise, we set $s_{1, i}=D_{d}$ and $\Delta v_{1, i}=v_{1}-v_{d}$ to let bus 1 traverse intersection $i$.

The condition for bus 1 stopping at signalized intersection $i$ during the yellow phase is given by equation (4):

$$
v_{1}^{2} /(2 b)<p_{s, i}-p_{1}-C_{\text {dist }}
$$


where $C_{\text {dist }}$ is a critical distance for detecting whether the bus near the intersection is able to stop upstream of the intersection.

State switching: If bus 1 moves downstream of signalized intersection $i$, then state $(1, i)$ will transfer to state 1 . Otherwise, state $(1, i)$ remains invariant.

\section{State 1: Speed recovery after traversing signalized intersection}

State action: Bus 1 follows a dummy predecessor, which is $D_{d}$ ahead of bus 1 , and runs at the speed of $\mathrm{v}_{d}$, that is $s_{1, i}=D_{d}$ and $\Delta v_{1, i}=v_{1}-v_{d}$.

State switching: If $p_{1} \geq k \cdot p_{s, i}+(1-k) \cdot p_{b, i}$, where $p_{b, i}$ is the position of stop $i$ and $k$ is a constant ranging from 0 to 1 , then state $(1, i)$ will transfer to state 2 . Otherwise, state $(1, i)$ remains invariant.

\section{State 2: Deceleration for approaching the stop}

State action: Bus 1 prepares to approach stop $i$ by setting $S_{1, i}=p_{b, i}+S_{\min }$ and $\Delta v_{1, i}=v_{1}$.

State switching: If $p_{1} \geq p_{b, i}$, then the current time is recorded to be the arrival time of bus 1 , and state $(1, i)$ transfers to state 3 . Otherwise, state $(1, i)$ remains invariant.

\section{State 3: Bus service at the stop}

State action: Bus 1 dwells at stop $i$ to offer bus service, that is $s_{1, i}=s_{\min }$ and $\Delta v_{1, i}=0$.

State switching: If $T D_{1, i}>T D_{c}$ with $T D_{1, i}$ denoting the time interval from the arrival time of bus 1 to the current time at stop $i$ and $T D_{c}$ a statistical service time, then the departure time of bus 1 is recorded by $d_{1, i}=t$, state $(1, i+1)$ activates as state 0 , and $i$ is updated to be $i+1$. Otherwise, state $(1, i)$ remains invariant.

\section{State 4: Other state}

State action: No action.

State switching: State $(1, i)$ transfers to state 0 if state $(1, i-1)$ transfers from state 3 to state 0 . Otherwise, state $(1, i)$ remains invariant.

\section{Variable Updating Process for the First Bus}

To update the dynamic motion of bus 1, we set $s_{h}=s_{1, i}$ and $\Delta v=\Delta v_{1, i}$, then according to equations (1) and (3) calculate the position, speed, and acceleration of bus 1 .

\section{Bus Propagation Model for Bus Followers}

The bus propagation model for each bus follower also includes a five-state machine and a variable updating process. The five states are defined as in Figure 1. The concrete state flow is given as follows.

\section{Five-State Machine for Bus Followers}

\section{State 0: Preparation for traversing the next signalized intersection}

State action: If bus $j$ is predicted to arrive at signalized intersection $i$ during the green phase, then it follows its predecessor (bus $j-1$ ) to go through intersection $i$, that is $s_{j, i}=p_{j-1}-p_{j}-L$ and $\Delta v_{j, i}=v_{j}-v_{j-1}$. If bus $j$ is predicted to arrive at signalized intersection $i$ during the red phase, then it has to stop upstream of the stop bar, that is $s_{j, i}=p_{s, i}-p_{j}$ and $\Delta v_{j, i}=v_{j}$. If bus $j$ is predicted to arrive at signalized intersection $i$ during the yellow phase and is able to stop before the stop line, meaning that bus $j$ satisfies condition (5), then we set $s_{j, i}=p_{s, i}-p_{j}$ and $\Delta v_{j, i}=v_{j}$. Otherwise, we set $s_{j, i}=p_{j-1}-p_{j}-L$ and $\Delta v_{j, i}=v_{j}-v_{j-1}$. 
The condition for bus $j$ stopping at signalized intersection $i$ is given by inequality (5).

$$
v_{j}^{2} /(2 b)<p_{s, i}-p_{1}-C_{d i s t}
$$

State switching: If bus $j$ moves downstream of signalized intersection $i$, that is $p_{j} \geq p_{s, i}$, then state $(j, i)$ transfers to state 1. Otherwise, state $(j, i)$ remains invariant.

\section{State 1: Speed recovery after traversing signalized intersection}

State action: Bus $j$ follows bus $j-1$ to drive ahead, that is $S_{j, i}=p_{j-1}-p_{j}-L$ and $\Delta v_{j, i}=v_{j}-v_{j-1}$.

State switching: If $p_{j} \geq k \cdot p_{s, i}+(1-k) p_{b, i}$ then state $(j, i)$ transfers to state 2 . Otherwise, state $(j, i)$ remains invariant.

\section{State 2: Deceleration for approaching the stop}

State action: Bus j prepares to approach stop $i$ by setting $s_{j, i}=p_{b, i}+S_{\min }$ and $\Delta v_{j, i}=v_{j}$.

State switching: If $p_{j} \geq p_{b i}$ or $p_{j-\mathrm{i}}-p_{j} \leq s_{\min }+\varepsilon_{s}$ and $v_{j} \leq \varepsilon_{v}$ with $\varepsilon_{s}$ and $\varepsilon_{v}$ small positive values, then the current time is recorded to be the arrival time of bus $j$ at stop $i$, and state $(j, i)$ transfers to state 3 . Otherwise, state $(j, i)$ remains invariant.

\section{State 3: Bus service at the stop}

To describe passenger swapping behavior, there are three kinds of bunching patterns to consider: no bus bunching, bunching with two buses, and bunching with three or more buses. To detect a bus bunching pattern, a five-length first-in-first-out queue is used to record the state of bus $j-2$, bus $j-1$, bus $j$, bus $j+1$, and bus $j+2$ with their latest stop number. The case to which the bus arrival pattern belongs is determined according to the state of the queue, and then the bus number to convert the new case is rolled into one of the three cases. The bus dynamic motion is also controlled by the state of the finite state machine. When the bus is going to depart from the stop, the bus dynamic motion and the state of the finite state machine should be updated at the same time.

\section{Case 1: No bus bunching}

If bus $j$ arrives at stop $i$ after bus $j-1$ departs the same stop, then the passenger boarding behavior can be described as shown by Figure 2.

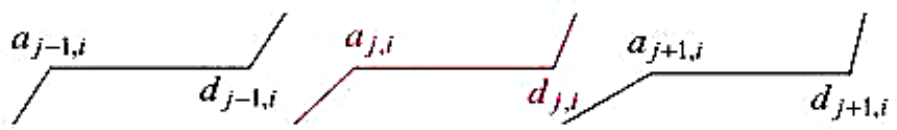

\section{FIGURE 2.}

No bus bunching, where $\mathrm{a}_{\mathrm{j}, \mathrm{i}}$ and $\mathrm{d}_{\mathrm{j}, \mathrm{i}}$ are bus $\mathrm{j}$ arrival and departure time from stop $\mathrm{i}$, respectively

State action - Case 1: Let $\lambda_{\mathrm{i}}$ be a uniform distribution variable to express the passenger arrival rate at stop $i$, then the number of passengers at stop $i$ waiting to board bus $j$ is given by equation (6):

$$
W_{j, i}=\left\lfloor\int_{d_{j-1, i}}^{a_{j, i}+T D_{j, i}} \lambda_{i} d t \mid+l_{j-1, i}\right.
$$


where $d_{j-1, i}$ is the departure time of bus $j-1$ from stop $i, T D_{j, i}$ denotes the time interval from the arrival time of bus $j$ at stop $i$ to the current time, $l_{j-1, i}$ is the number of leftover passengers by bus $j-1$ at stop $i$, and $[\cdot]$ is a downward rectification operator.

According to assumption 9, the alighting demand of bus $j$ at stop $i$ is given by equation (7):

$$
A_{j, i}=\operatorname{round}\left(\rho_{i} . L_{j, i-1}\right)
$$

where $L_{j, i-1}$ is the number of onboard passengers of bus $j$ between stop $i-1$ and $i, \rho_{i}$ is the alighting proportion at stop $i$, and round $(\cdot)$ is an operator rounding to the nearest integer.

Let $b_{j, i}$ be the boarding rate for bus $j$ at stop $i$, then the number of actual boarding passengers is limited by the competition between the number waiting and the capacity of bus $j$, given by equation (8):

$$
B_{j, i}=\min \left(\left\lfloor\int_{a_{j, i}}^{a_{j, i}+T D_{j, i}} b_{j, i} d t\right\rfloor, W_{j, i}, C_{j}-L_{j, i-1}+A_{j, i}\right)
$$

where $C_{j}$ is the capacity of bus $j$.

The number of onboarding passengers of bus $j$ between stop $i$ and $i+1$ given by equation (9) is the sum of the number of onboarding passengers of bus $j$ between stop $i-1$ and stop $i$, and the difference between the number of actual boarding passengers and alighting passengers.

$$
L_{j, i}=L_{j, i-1}-A_{j, i}+B_{j, i}
$$

The number of leftover passengers by bus $j$ at stop $i$ is the difference between the number of waiting passengers and actual boarding passengers, that is $l_{j, i}=\max \left(W_{j, i}-B_{j, i}, 0\right)$.

State switching - Case 1: If all passengers at stop $i$ have boarded bus $j$, that is $W_{j, i} \leq B_{j, i}$, or the capacity of bus $j$ has been reached, that is $B_{j, i} \geq C_{j}-L_{j, i-1}+A_{j, i}$, then if the dwell time of bus $j$ at stop $i$ is more than $T D_{c, j, i}=A_{j, i} \cdot a_{j, i}$ where $a_{j, i}$ is the alighting rate for bus $j$ at stop $i$, then the current time is recorded to be the departure time of bus $j$ from stop $i$, that is $d_{j, i}=t$, then state $(j, i+1)$ transfers to state 0 , and $i$ is updated to be $i+1$. Otherwise, state $(j, i)$ remains invariant.

\section{Case 2: Bunching with two buses}

If bus $j$ arrives at stop $i$ before bus $j-1$ departs the same stop, then the boarding behavior is described as shown in Figure 3.

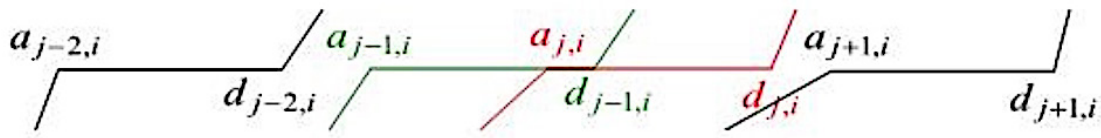

\section{FIGURE 3.}

Bunching with two buses 
State action - Case 2: Suppose each stop is equipped with a passenger service system, which senses the number of passengers at the station and can automatically decide and display the number of passengers to board the front and rear of the bus, respectively. Therefore, when two buses bunch, the passenger service system will automatically guide passengers to form two groups to board the bunching buses. This paper assumes a constant swapping rate $p s r$, then when bus $j-1$ and $j$ serve stop $i$, the number of remaining waiting passengers at stop $i$ is given by equation (10) when bus $j$ arrives at stop $i$ :

$$
W_{j-1, i}^{r}=\left\lfloor\int_{d_{j-2, i}}^{a_{j-1, i}+T D_{j-1, i}} \lambda_{i} d t\right\rfloor+l_{j-2, i}-B_{j-1, i}^{1}
$$

with $\left.B_{j-1, i}^{1}=\min \left(\mid \int_{a_{j-1, i}}^{a_{j, i}} b_{j-1, i} d t\right],\left|\int_{d_{j-2, i}}^{a_{j, i}} \lambda_{i} d t\right|+l_{j-2, i}\right)$ the number of passengers who have boarded bus $j-1$ before $a_{j, i}$. Here, we address the time when bus $j-1$ will depart from stop $i$ because after that we will judge the bunching pattern again and then decide the time when the succeeding bus will depart from stop $i$ in an iterative way.

To ensure bus $j-1$ departs stop i prior to bus $j$, the passenger service system should use a small passenger swapping rate ( $p s r)$ to command remaining waiting passengers to board at the rear of the bus. Then the number of passengers waiting to board bus $j-1$ and the number of actual boarding passengers can be given by equations (11) and (12), respectively.

$$
\begin{gathered}
W_{j-1, i}=B_{j-1, i}^{1}+p s r \cdot W_{j-1, i}^{r} \\
B_{j-1, i}=\min \left(\left\lfloor\int_{a_{j-1, i}}^{a_{j-1, i}+T D_{j-1, i}} b_{j-1, i} d t\right\rfloor, W_{j-1, i}, C_{j-1}-L_{j-1, i-1}+A_{j-1, i}\right)
\end{gathered}
$$

State switching - Case 2: As for bus $j-1$, if bus $j-1$ is still at stop $i$, and $B_{j-1, i} \geq \min \left(W_{j-1, i}, C_{j-1}-L_{j-1, i-1},+\mathrm{A}_{j-1, i}\right)$, and the dwell time of bus $j-1$ at stop $i$ is more than $T D_{c, j-1, i}$, then $\left.d_{j-1, i}=t, l_{j-1, i}=\max \left(W_{j-1}^{i}\right)+B_{j-1, i}^{1}-B_{j-1, i}, 0\right), L_{j-1 i}=$ $L_{j-1, i-1}-A_{j-1, i}+B_{j-1}$, state $(j-1, i+1)$ transfers to state 0 , and $i$ is updated by $i+1$. Otherwise, state $(j-1, i)$ remains invariant, and the number of leftover and onboarding passengers do not need to update, respectively.

REMARK 2. Passenger service system is a future device for bus stops. Currently, some stops establish some guardrails and electronic screens to guide passengers waiting in line and provide next bus information, as is observed in the Hangzhou Railway eastern station bus stop in China. The passenger service system function can be implemented by these facilities and customer service representatives, enabling them to swap remaining passengers between the front bunching bus and rear one under the supervision of agency staff.

\section{Case 3: Bunching with three or more buses}

If bus $j$ arrives at stop $i$ before bus $j-2$ departs stop $i$, then the bus motion is given as shown in Figure 4 .
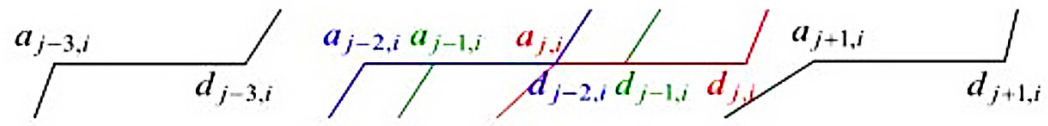

FIGURE 4.

Bunching with three or more buses 
State action - Case 3: Bus $j-2$ leaves stop $i$ when it finishes unloading passengers, on the condition that bus $j$ has already arrived at stop $i$. In this case, the number of passengers waiting for bus $j-2$ at stop $i$ is given by equation (13), and the number of actual boarding passengers of bus $j-2$ at stop $i$ is given by equation (14).

$$
\begin{gathered}
W_{j-2, i}=\left\lfloor\int_{d_{j-3, i}}^{a_{j-2, i}+T D_{j-2, i}} \lambda_{i} d t\right\rfloor+l_{j-3, i} \\
B_{j-2, i}=\min \left(\left\lfloor\int_{a_{j-2, i}}^{a_{j-2, i}+T D_{j-2, i}} b_{j-2, i} d t\right\rfloor,\left\lfloor\int_{d_{j-3, i}}^{a_{j-2, i}+T D_{j-2, i}} \lambda_{i} d t\right\rfloor+l_{j-3, i}, C_{j-2}-L_{j-2, i-1}+A_{j-2, i}\right)
\end{gathered}
$$

The number of onboarding passengers of bus $j-2$ between stop $i$ and $i+1$ can be updated by $L_{j-2, i}=L_{j-2, i-1}-A_{j-2, i}$ $+B_{j-2, i}$ and the number of leftover passengers by bus $j-2$ at stop $i$ is given by $l_{j-2, i}=\max \left(W_{j-2, i}-B_{j-2, i} 0\right)$.

Similar to Case 2, the time when bus j-2 departs stop $i$ must be addressed, and then the time when a succeeding bus departs stop $i$ can be iteratively determined.

State switching - Case 3: As for bus $j-2$, if bus $j-2$ finishes unloading passengers after bus $j$ has already reached stop $i$, then state $(j-2, i+1)$ transfers to state 0 , and $i$ is updated by $i+1$. Otherwise, state $(j-2, i)$ remains invariant.

REMARK 3. Since an iterative method is used to implement passenger assignment, three or more buses bunching can be treated to be the same as Case 3 .

\section{State 4: Other state}

State action: No action.

State switching: State $(j, i)$ transfers to state 1 if state $(j, i-1)$ transfers from state 3 to state 0 . Otherwise, state $(j, i)$ remains invariant.

\section{Variable Updating Process for Bus Followers}

To update the dynamic motion of bus $j$, we set $s_{h}=s_{j, j}, \Delta v=\Delta v_{j, j}$, then according to equations (1) and (3) calculate the position, speed, and acceleration of bus $j$.

Table 1 shows how to determine to which bunching pattern bus $j$ belongs, where "I" means there is no need to consider. Take $j$ ranges from 3 to $N-2$ ( $N$ denotes the total number of buses) for example. If the bus queue state is (Depart, Depart, Dwell, Not yet arrive, Not yet arrive), then "no bus bunching" is used to simulate the bus service at the stop. If the bus queue state is (Depart, Dwell, Dwell, Not yet arrive, Not yet arrive), then "bunching with 2 buses" is used to simulate the bus service at the stop. If the bus queue is (Depart, Depart, Dwell, Dwell, Not yet arrive), then we need to roll $j$ to $j+1$ and use "bunching with 2 buses" to simulate the bus service at the stop; otherwise, we need to roll $j$ forward or backward to $j_{n e w}$ correspondingly, and then use "bunching with 3 buses" to simulate the bus service at the stop. 
TABLE 1.

Five-Length Queue Based Bunching Pattern Classification

\begin{tabular}{|c|c|c|c|c|c|c|c|}
\hline \multirow{2}{*}{$\begin{array}{c}\text { Bus } \\
\text { Number }\end{array}$} & \multicolumn{5}{|c|}{ Bus Queue State } & \multirow{2}{*}{$\begin{array}{l}\text { Bus Bunching } \\
\text { Pattern }\end{array}$} & \multirow{2}{*}{$\begin{array}{l}\text { Rolling } \\
\text { Method }\end{array}$} \\
\hline & Bus $j-2$ & Bus $j-1$ & Bus $j$ & Bus $j+1$ & Bus $j+2$ & & \\
\hline \multirow{3}{*}{$j=1$} & 1 & 1 & Dwell & Not yet & Not yet & No bus bunching & $j_{\text {new }}=j$ \\
\hline & 1 & 1 & Dwell & Dwell & Not yet & Bunching with 2 buses & $j_{\text {new }}=j+1$ \\
\hline & 1 & 1 & Dwell & Dwell & Dwell & Bunching with 3 buses & $j_{\text {new }}=j+2$ \\
\hline \multirow{5}{*}{$j=2$} & 1 & Depart & Dwell & Not yet & Not yet & No bus bunching & $j_{\text {new }}=j$ \\
\hline & 1 & Dwell & Dwell & Not yet & Not yet & Bunching with 2 buses & $j_{\text {new }}=j$ \\
\hline & 1 & Depart & Dwell & Dwell & Not yet & Bunching with 2 buses & $j_{\text {new }}=j+1$ \\
\hline & 1 & Dwell & Dwell & Dwell & Not yet & Bunching with 3 buses & $j_{\text {new }}=j+1$ \\
\hline & 1 & Depart & Dwell & Dwell & Dwell & Bunching with 3 buses & $j_{n e w}=j+2$ \\
\hline \multirow{6}{*}{$2<j<N-1$} & Depart & Depart & Dwell & Not yet & Not yet & No bus bunching & $j_{\text {new }}=j$ \\
\hline & Depart & Dwell & Dwell & Not yet & Not yet & Bunching with 2 buses & $j_{\text {new }}=j$ \\
\hline & Depart & Depart & Dwell & Dwell & Not yet & Bunching with 2 buses & $j_{n e w}=j+1$ \\
\hline & Dwell & Dwell & Dwell & Not yet & Not yet & Bunching with 3 buses & $j_{\text {new }}=j$ \\
\hline & Depart & Dwell & Dwell & Dwell & Not yet & Bunching with 3 buses & $j_{\text {new }}=j+1$ \\
\hline & Depart & Depart & Dwell & Dwell & Dwell & Bunching with 3 buses & $j_{\text {new }}=j+2$ \\
\hline \multirow{5}{*}{$j=N-1$} & Depart & Depart & Dwell & Not yet & 1 & No bus bunching & $j_{\text {new }}=j$ \\
\hline & Depart & Dwell & Dwell & Not yet & 1 & Bunching with 2 buses & $j_{\text {new }}=j$ \\
\hline & Depart & Depart & Dwell & Dwell & 1 & Bunching with 2 buses & $j_{\text {new }}=j+1$ \\
\hline & Dwell & Dwell & Dwell & Not yet & 1 & Bunching with 3 buses & $j_{\text {new }}=j$ \\
\hline & Depart & Dwell & Dwell & Dwell & 1 & Bunching with 3 buses & $j_{n e w}=j+1$ \\
\hline \multirow{3}{*}{$j=N$} & Depart & Depart & Dwell & 1 & 1 & No bus bunching & $j_{\text {new }}=j$ \\
\hline & Depart & Dwell & Dwell & 1 & 1 & Bunching with 2 buses & $j_{\text {new }}=j$ \\
\hline & Dwell & Dwell & Dwell & 1 & 1 & Bunching with 3 buses & $j_{\text {new }}=j$ \\
\hline
\end{tabular}

\section{Connected Bus Propagation Model}

Due to demand variation and unexpected traffic signals, it is difficult to simultaneously improve the energy efficiency and service reliability of a human-driven bus. Thanks to the wide application of V2X communication, buses can be made smarter by equipping them with a V2X communication device and signal controller. Connected buses can share position and speed with other buses and can receive signal phasing and timing (SPaT) information. This paper aims to improve the energy efficiency and service reliability for the connected bus, and attempts to use the arrival time schedule, departure time, and next signal controller SPaT information to plan an anticipated average speed for the connected bus. Then, MPC can be used to generate a smooth trajectory to guide the connected bus to traverse signalized intersections without stopping and reach the next stop with fewer delays. 


\section{Anticipated Average Speed Planning (AASP)}

With a proper value of passenger swapping rate ( $p s r)$, the front bunching bus departs the stop earlier, while the rear one departs later. However, this state is so weak that these two buses will bunch again after one or more stops. Holding control is an efficient way to adjust bus departure time to avoid bunching, but this method cannot work well if the bus always encounters unexpected traffic signals. To overcome this problem, AASP is used to allow the bus to arrive at the next stop at the scheduled time and arrive at the next intersection during a green phase.

First, the crossing point $t_{t l}$ of the next intersection $i+1$ between $\left(d_{j, i}, p_{b, i}\right)$ and $\left(a_{j, i+1}\right.$ schedul $\left.p_{b, i+1}\right)$ is given by equation (15), where $a_{j, i+1}^{\text {schedule }}$ is the scheduled arrival time of bus $j$ at stop $i+1$.

$$
t_{t l}=d_{j, i}+\frac{a_{j, i+1}^{s c h e d u l e}-d_{j, i}}{p_{b, i+1}-p_{b, i}}\left(p_{s, i+1}-p_{b, i}\right)
$$

If $t_{t l}$ falls during the green phase, then bus $j$ can run at a constant speed to traverse the signalized intersection $i+1$ and arrive at stop $i+1$ at the scheduled time $a_{j, i+1}^{\text {schedule }}$. In this case, the anticipated average speed $v_{j}^{i d}$ can be derived by equation (16).

$$
v_{j}^{i d}=\left(p_{b, i+1}-p_{b, i}\right) /\left(a_{j, i+1}^{\text {schedule }}-d_{j, i}\right)
$$

Otherwise, Table 2 must be referenced for a suitable decision and the corresponding anticipated average speed, where the AASP decision (DEC) is given by Figure 5. For example, when bus $j$ departs stop $i$ (current stop number) during the red phase, and bus $j$ is expected to arrive at stop $i+1$ during the green phase of the current cycle, then if bus $j$ is predicted to arrive at signalized intersection $i+1$ during the green phase, it will adopt a constant speed to reach stop $i+1$. Otherwise, bus $j$ has to regulate its speed to arrive at intersection $i+1$ at the beginning of the green phase of the next cycle, and bus $j$ may delay if it cannot reach stop $i+1$ at its maximum speed, indicating that bus $j$ can travel at speed of $v_{j}^{i d}=\left(p_{j+1, t l}-p_{j, s}\right) /\left(t_{\text {rem }}+T_{R}\right)$ upstream of intersection $i+1$ where $t_{\text {rem }}$ is the remaining time of the signal and $T_{R}$ is the red phase duration, and at the speed of $v_{j}^{i d}=\min \left(v_{\max }\left(p_{j+1, s}-p_{j+1, t)}\right) /\left(a_{j, i+1}^{\text {schedule }}-d_{j, i}-t_{r e m}-T_{R}\right)\right)$ downstream of intersection $i+1$. The decision and anticipated average speed for other cases can be obtained similarly by referring to Table 2. 
Modeling Bus Bunching and Anti-bunching Control Accounting for Signal Control and Passenger Swapping Behavior

TABLE 2.

AASP to Allow Bus to Arrive at Next Stop Reliably and with Energy Efficiency

\begin{tabular}{|c|c|c|c|c|c|}
\hline CSDT & NSAST & USIOCT & $\begin{array}{c}\text { AASP } \\
\text { Decision }\end{array}$ & Anticipated Average Speed & Result \\
\hline \multirow{6}{*}{ CRL } & CRL & CRL & DEC-1 & $\begin{array}{l}\text { USI: }\left(p_{s, i+1}-p_{b, i}\right) / t_{r e m} \\
\text { DSI: } v_{\max }\end{array}$ & Delay \\
\hline & \multirow[t]{2}{*}{ CGL } & CRL & DEC-2 & $\begin{array}{l}\text { USI: }\left(p_{s, i+1}-p_{b, i}\right) / t_{r e m} \\
\text { DSI: } \min \left(v_{\max }, \frac{p_{j+1, s}-p_{j+1, t l}}{a_{j, i+1}^{\text {schedule }}-d_{j, i}-t_{r e m}-T_{R}}\right)\end{array}$ & Maybe delay \\
\hline & & CGL & DEC-3 & $\left(p_{b, i+1}-p_{b, i}\right) /\left(a_{j, i+1}^{\text {schedule }}-d_{j, i}\right)$ & Reliable \\
\hline & \multirow{3}{*}{ NRL } & \multirow{2}{*}{ NRL } & \multirow{2}{*}{ DEC-4 } & $\begin{array}{l}\text { USI: } \quad\left(p_{s, i+1}-p_{b, i}\right) /\left(t_{r e m}+T_{G}\right) \\
\text { DSI: } \frac{p_{b, i+1}-p_{s, i+1}}{a_{j, i+1}^{\text {schedule }}-d_{j, i}-t_{r e m}-T_{G}}\end{array}$ & $\begin{array}{l}\text { Accelerating to } \\
\text { cross: reliable }\end{array}$ \\
\hline & & & & $\begin{array}{l}\text { USI: }\left(p_{s, i+1}-p_{b, i}\right) /\left(t_{r e m}+T_{G}+T_{R}\right) \\
\text { DSI: } v_{\max }\end{array}$ & $\begin{array}{l}\text { Slow down to } \\
\text { cross: delay }\end{array}$ \\
\hline & & CGL & DEC-5 & $\left(p_{b, i+1}-p_{b, i}\right) /\left(a_{j, i+1}^{\text {schedule }}-d_{j, i}\right)$ & Reliable \\
\hline \multirow{6}{*}{ CGL } & CGL & CGL & DEC-6 & $\left(p_{b, i+1}-p_{b, i}\right) /\left(a_{j, i+1}^{\text {schedule }}-d_{j, i}\right)$ & Reliable \\
\hline & \multirow{3}{*}{ CRL } & CGL & DEC-7 & $\left(p_{b, i+1}-p_{b, i}\right) /\left(a_{j, i+1}^{\text {schedule }}-d_{j, i}\right)$ & Reliable \\
\hline & & \multirow[b]{2}{*}{ CRL } & \multirow[b]{2}{*}{ DEC-8 } & $\begin{array}{l}\text { USI: }\left(p_{s, i+1}-p_{b, i}\right) / t_{r e m} \\
\text { DSI: }\left(p_{b, i+1}-p_{s, i+1}\right) /\left(a_{j, i+1}^{\text {schedule }}-d_{j, i}-t_{r e m}\right)\end{array}$ & $\begin{array}{l}\text { Accelerating to } \\
\text { cross: reliable }\end{array}$ \\
\hline & & & & $\begin{array}{l}\text { USI: }\left(p_{s, i+1}-p_{b, i}\right) /\left(t_{r e m}+T_{R}\right) \\
\text { DSI: } v_{\max }\end{array}$ & $\begin{array}{l}\text { Slow down to } \\
\text { cross: maybe } \\
\text { delay }\end{array}$ \\
\hline & \multirow[b]{2}{*}{ NGL } & NGL & DEC-9 & $\left(p_{b, i+1}-p_{b, i}\right) /\left(a_{j, i+1}^{\text {schedule }}-d_{j, i}\right)$ & Reliable \\
\hline & & CRL & DEC-10 & $\begin{array}{l}\text { USI: }\left(p_{s, i+1}-p_{b, i}\right) /\left(t_{r e m}+T_{R}\right) \\
\text { DSI: } \min \left(v_{\max }, \frac{p_{j+1, s}-p_{j+1, t l}}{a_{j, i+1}^{\text {schedule }}-d_{j, i}-t_{r e m}-T_{R}}\right)\end{array}$ & Maybe delay \\
\hline
\end{tabular}

CSDT = current stop departure time, NSAST = next stop arrival schedule time, USIOCT = next signalized intersection original crossing time, $C R L=$ current red signal, $N R L=$ next red signal, $C G L=$ current green signal, $N G L=$ next green signal, USI = upstream of signalized intersection, $D S I=$ downstream of signalized intersection, and $T_{G}=$ green signal duration . 


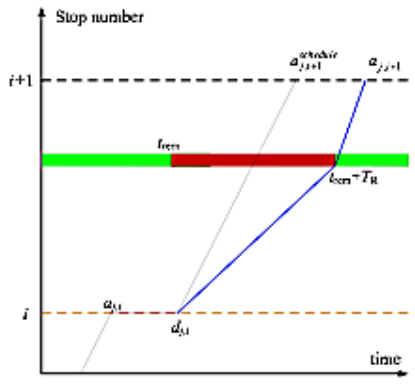

(a) DEC-1

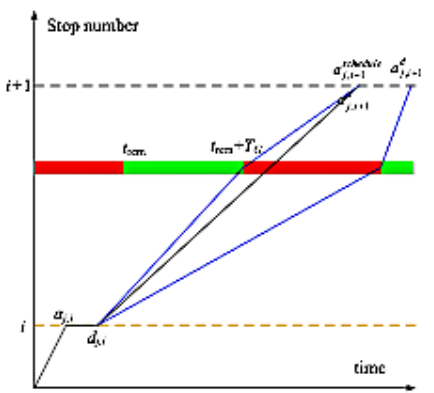

(d) DEC-4

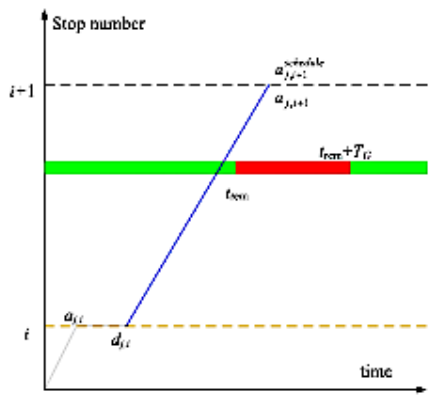

(g) DEC-7

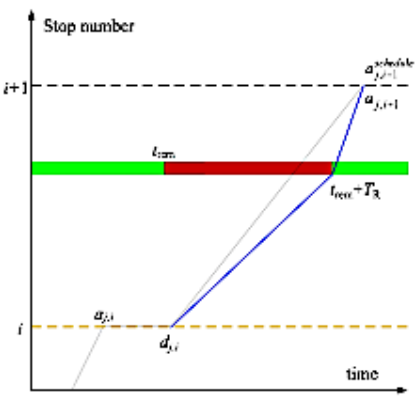

(b) DEC-2

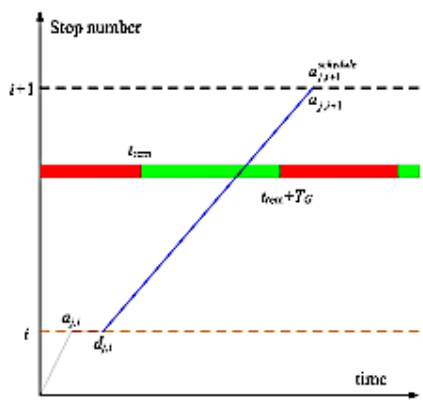

(e) DEC-5

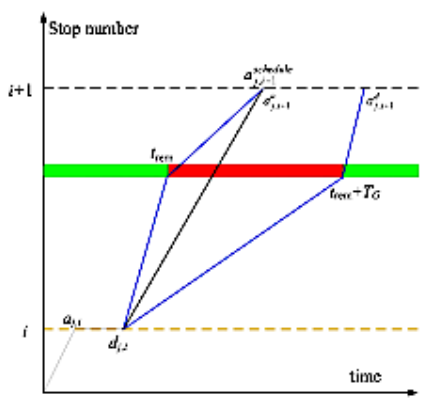

(h) DEC-8

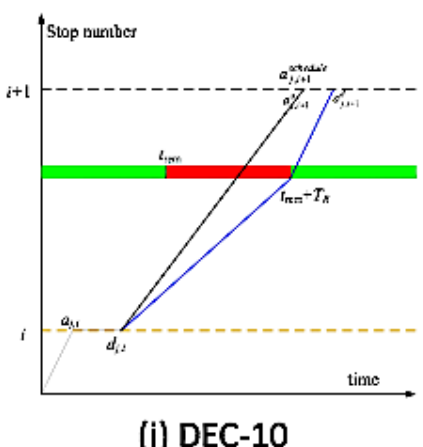

(j) DEC-10

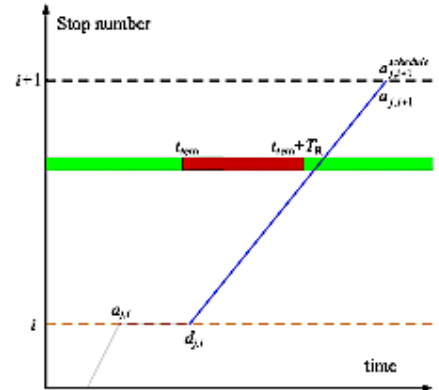

(c) DEC-3

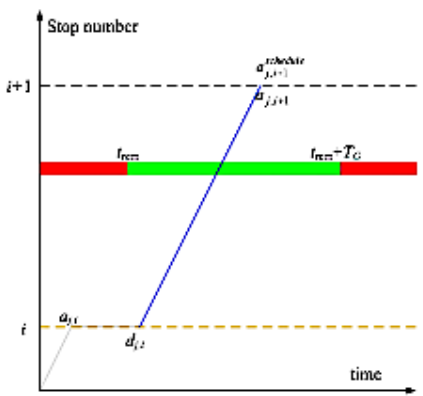

(f) DEC-6

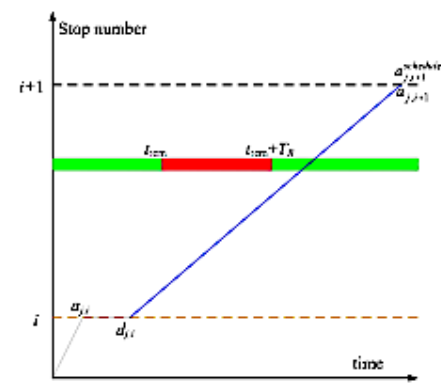

(i) DEC-9

\section{FIGURE 5.}

AASP decisions for various combinations of CSDT, NSAST, and USIOCT 
REMARK 4. Table 2 and Figure 5 can be extended to consider the remaining signal phase of the next signal cycle and the signal phase of the signal cycle after that.

REMARK 5. If $a_{j, i+1}^{\text {schedele }}<d_{t, j}$, then bus $j$ needs to travel at its maximum speed both upstream and downstream of signalized intersection $i+1$ to reduce delays.

\section{Smooth Trajectory Generated Based on MPC}

To generate a smooth trajectory to follow the anticipated average speed, the motion equation for connected bus $j$ is given by equation (17):

$$
x_{j, k}=F \cdot x_{j, k-1}+G \cdot u_{j, k}
$$

where $T$ is the time updating step, $x_{j, k}=\left[p_{j, k} v_{j, k}\right]^{T}$ is the location and speed vector of bus $j$ at time point $k T$, and $u_{j, k}$ is the acceleration of bus $j$ at time point $k T$. The system matrix $F$ and input matrix $G$ are given by equation (18).

$$
F=\left[\begin{array}{ll}
1 & T \\
0 & 1
\end{array}\right], G=\left[\begin{array}{c}
T^{2} / 2 \\
T
\end{array}\right]
$$

Let $N c$ be the number of predicting step, and let $X_{j, k}=\left[x_{j, k+1}^{\mathrm{T}}, x_{j, k+2}^{\mathrm{T}}, \cdots, x_{j, k+N c}^{\mathrm{T}}\right]^{\mathrm{T}}$ and $U_{j, k}=\left[U_{j, k+1}, u_{j, k+2}, \cdots, u_{j, k+N c}\right]^{\mathrm{T}}$, then the subsequent system states can be derived by equation (19):

$$
X_{j, k}=\Phi U_{j, k}+\Gamma x_{j, k}
$$

with the coefficient matrices given by equation (20).

$$
\Phi=\left[\begin{array}{ccccc}
G & 0 & 0 & 0 & 0 \\
F G & G & 0 & 0 & 0 \\
F^{2} G & F G & G & 0 & 0 \\
\vdots & \vdots & \ddots & G & 0 \\
F^{N c-1} G & F^{N c-2} G & \cdots & \cdots & G
\end{array}\right], \Gamma=\left[\begin{array}{c}
F \\
F^{2} \\
F^{3} \\
\vdots \\
F^{N c}
\end{array}\right]
$$

Let $X_{j, k}^{d}=\left[p_{j, k}+v_{j}^{i d} T, v_{j}^{i d}, p_{j, k}+2 \cdot v_{j}^{i d} T, v_{j}^{i d}, p_{j, k}+3 \cdot v_{j}^{i d} T, v_{j}^{i d}, \cdots, p_{j, k}+N c \cdot v_{j}^{i d} T, v_{j}^{i d}\right]^{T}$ and define the state errors as $\tilde{X}_{j, k}=X_{j, k}-X_{j, k}^{d}$, then we can obtain the state error dynamics from equation (21).

$$
\tilde{X}_{j, k}=\Phi U_{j, k}+\Gamma x_{j, k}-X_{j, k}^{d}
$$

To obtain the solution of $\boldsymbol{U}_{i, k}, M P C$ is used. First, the cost function for connected bus $j$ approaching intersection $i$ is given as $J_{j, i}=\tilde{X}_{j, k}^{\mathrm{T}} Q \tilde{X}_{j, k} / 2+U_{j, k}^{\mathrm{T}} R U_{j, k} / 2$, then equation (21) is substituted into the cost function to obtain the cost function shown by equation (22):

$$
J_{j, i}=U_{j, k}^{T} D_{1} U_{j, k} / 2+D_{2} U_{j, k}+D_{3}
$$


with the coefficient matrices given by equation (23)

$$
D_{1}=\Phi^{T} Q \Phi+R, \quad D_{2}=\left(\Gamma x_{j, k}-X_{j, k}^{d}\right)^{T} Q \Phi
$$

subject to the input constraints in equation (24)

$$
M U_{j, k} \leq \Theta
$$

with the coefficient matrices given by equation (25)

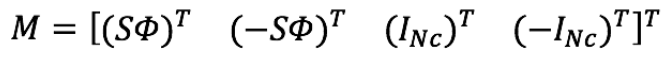

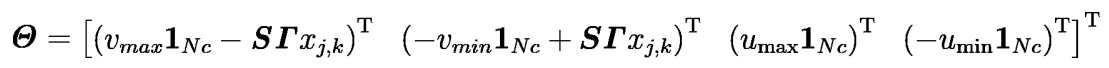

where $I_{N C}$ is an identity matrix with $N C \times N C$ dimension; $1_{N C}$ is a row vector with $N_{c}$ elements 1 ; and $S$ is the Kronecker product between $I_{N C}$ and [0 1$]$.

Then the Primal-Dual method can be used to solve the original optimal control problem (22)-(25), which is equivalent to minimizing the dual objective function in equation (26):

$$
J_{j, i}=\lambda^{T} \Psi_{1} \lambda / 2+\lambda^{T} \Psi_{2}+\Theta^{T} D_{1}^{-1} \Theta / 2
$$

where the coefficient matrices are given by equation (27)

$$
\Psi_{1}=M D_{1}^{-1} M^{T}, \Psi_{2}=\Theta+M D_{1}^{-1} D_{2}
$$

subject to $\lambda \geq 0$. The active set of $\lambda$ is denoted as $\lambda_{a c t}$ and the corresponding constraints are described by $M_{a c t}$ and $\Theta_{a c t}$, then the optimal accelerations are given by equation (28).

$$
U_{j, k}=-D_{1}^{-1} D_{2}-D_{1}^{-1} M_{a c t}^{T} \lambda_{a c t}
$$

$\lambda_{\text {act }}$ can be calculated by Hildreth's quadratic programming procedure, which is given by equation (29):

$$
\lambda_{l}^{m+1}=\max \left(0,-\left(\psi_{2, l}+\sum_{k=1}^{l-1} \psi_{1, l k} \lambda_{k}^{m+1}+\sum_{k=l+1}^{N} \psi_{1, l k} \lambda_{k}^{m}\right) / \psi_{1, l l}\right)
$$

where $\Psi_{1, l k}$ is the th $^{\text {th }}$ row and $k^{\text {th }}$ column element of $\Psi_{1}, \psi_{2, l}$ is the $l^{\text {th }}$ row element of $\Psi_{2}$. If $\lambda^{m+1}$ is sufficient close to $\lambda^{m}$, we deem that $\lambda^{m}$ is the numerical solution for $\lambda$, then $\lambda_{\text {act }}$ is the nonzero set of $\lambda$, and $M_{\text {act }}$ is the corresponding section of $M$ according to the nonzero set of $\lambda$. When $\lambda_{\text {act }}$ and $M_{\text {act }}$ are determined, the control input can be derived according to equation (28).

\section{Simulation}

To simplify the numerical simulations and related performance comparisons, we assume all buses have similar characteristics; all bus stops are constructed the same and located on the far side of the signalized intersection; 
and all signal controllers have the same red, green, and yellow phase duration. The differences focus on the initial location, speed of the bus, and stochastic passenger arrival pattern.

\section{Associated Performance Metrics}

Eight performance metrics are used to calculate the benefits of the proposed model.

1. Mean value of headways (M-TH)/standard deviation of headways (STD-TH): the mean value/ standard deviation of the difference between two successive buses' departure times.

2. Bunching rate (BR): the ratio of bunching times to the product of bus number and stop number. Bus bunching occurs when the headway is greater than $1.5 \times \mathrm{M}-\mathrm{TH}$ or less than $0.5 \times \mathrm{M}-\mathrm{TH}$.

3. Mean value of dwell time (M-DT): the mean value of the difference between bus departure time and arrival time.

4. Mean value of waiting time for green signal (M-WTGL)/standard deviation of waiting time for green signal (STD-WTGL): the mean value/standard deviation of the difference between the green signal starting time and the time of the bus arriving at the stop bar of the signalized intersection.

5. Travel time per kilometer (TTPK): the ratio of the mean value of the travel time to $s_{\text {end }}$, where $s_{\text {end }}=$ $500 \times$ (stop number) +500 .

6. Passenger waiting time (PWT): the mean value of the waiting time for the newcomer and the leftovers. The calculation formula can turn to Wu et al. (2017).

7. Average load factor (ALF): the ratio of the number of onboard passengers to the product of the bus number and stop number.

8. Fuel Efficiency (FE): the Virginia Tech Comprehensive Power-based Fuel consumption Model (VT CPFM, Wang and Rakha 2016) is used to calculate fuel efficiency. The fuel consumption rate of bus $j$ can be estimated by equations (30)-(32):

$$
\begin{gathered}
F C_{j}(k)=\left\{\begin{array}{cc}
\alpha_{0}+\alpha_{1} \cdot P_{j}(k)+\alpha_{2} \cdot P_{j}^{2}(k) & P_{j}(k) \geq 0 \\
\alpha_{0} & P_{j}(k)<0
\end{array}\right. \\
P_{j}(k)=\frac{R_{j}(k)+1 \cdot 1 \cdot\left(m_{b}+L_{j, i}(k) m_{p}\right) \cdot a_{j}(k)}{1000 \cdot \eta_{d}} \cdot v_{j}(k) \\
R_{j}(k)=\rho C_{d} C_{h} A_{f} v_{j}^{2}(k) / 2+\left(m_{b}+L_{j, i}(k) m_{p}\right) g \frac{C_{r 0}}{1000}\left(3.6 \cdot C_{r 1} v_{j}(k)+C_{r 2}\right)
\end{gathered}
$$

where $\alpha_{0}, \alpha_{1}, \alpha_{2}$ are model constants determined by bus configuration; $P_{j}(k)$ is the instantaneous power of bus $j$ at time point $k T ; m_{b}$ is the bus mass; $m_{p}$ is the average mass of passenger; $a_{j}(k)$ is the acceleration rate of bus $j$ at time point $k T ; \eta_{d}$ is the driveline efficiency; $R_{j}(k)$ is the resistance force at time point $k T$ for bus $j ; \rho$ is the air density at sea level at the temperature of $15^{\circ} \mathrm{C}$; $C_{d}$ is the drag coefficient of the bus; $C_{h}$ is the altitude correction factor; and $A_{f}$ is the frontal area of the bus. The rolling resistance constants are $C_{r 0}, C_{r 1}, C_{r 2}$, respectively, and the gravitational constant is $g$. 


\section{Simulation Settings}

Numerical simulations for both the human-driven and connected bus propagation models are carried out by using the fourth-order Runge-Kutta method at the updating frequency of 2 hertz. The simulation focuses on frequency-based bus operations, and the initial states of the buses are given by equation (33):

$$
\left\{\begin{array}{l}
v_{j}(0)=v_{i n i}+\omega_{v j}, a_{j}(0)=0, \operatorname{gap}_{j}(0)=s_{m i n}+S F \cdot T_{s} \cdot v_{j}(0)+\omega_{p j} \\
p_{1}(0)=0, p_{j}(0)=p_{1}(0)-\sum_{l l=2}^{j} g a p_{l l}(0) \\
E \omega_{v j}=0, E \omega_{p j}=0, E \omega_{v j} \cdot \omega_{v j}=R_{v j}, E \omega_{p j} \cdot \omega_{p j}=R_{p j}
\end{array}\right.
$$

where $v_{i n i}=6.94 \mathrm{~m} / \mathrm{s}, R_{v j}=1 \mathrm{~m}^{2} / \mathrm{s}^{2}$, and $R_{p j}=1 \mathrm{~m}^{2}$ are the variances for normal random variables $\omega_{v j}$ and $\omega_{p j}$, respectively. The case $p_{j}$ is greater than 0 , indicating that bus $j$ departs the bus terminal. The minimum bus speed is set as $0 \mathrm{~km} / \mathrm{h}$ and the maximum is set at $50 \mathrm{~km} / \mathrm{h}$. $S F$ is a parameter that equals $3600 /$ (service frequency)/ $T_{s}$. The total bus number $\mathrm{N}$ considered in the simulation is 16 .

The traffic signal controller configurations for all intersections are 120 second (s) cycle length; 0.6 red ratio; and $3 \mathrm{~s}$ yellow phase. The initial traffic signal controller states for each signalized intersection are given by Table 3. When cycle length changes, the current phase does not change, and remaining time will be updated by the modulus after the division of the remaining time in Table 3 by the corresponding duration. Bus stops are located 100 meters $(\mathrm{m})$ downstream of the signalized intersection. Table 4 gives the parameters of the intelligent driver model (IDM) and VT-CPFM model.

\section{TABLE 3.}

Initial States of Traffic Signal Controller

\begin{tabular}{cccc|cccc}
\hline Number & $\begin{array}{c}\text { Position } \\
(\mathbf{m})\end{array}$ & $\begin{array}{c}\text { Remaining } \\
\text { Time (s) }\end{array}$ & $\begin{array}{c}\text { Current } \\
\text { Phase }\end{array}$ & Number & $\begin{array}{c}\text { Position } \\
(\mathbf{m})\end{array}$ & $\begin{array}{c}\text { Remaining } \\
\text { Time }(\mathbf{s})\end{array}$ & $\begin{array}{c}\text { Current } \\
\text { Phase }\end{array}$ \\
\hline 1 & 400 & 7 & Green & 9 & 4400 & 15 & Red \\
2 & 900 & 35 & Red & 10 & 4900 & 22 & Green \\
3 & 1400 & 27 & Green & 11 & 5400 & 42 & Green \\
4 & 1900 & 55 & Red & 12 & 5900 & 5 & Red \\
5 & 2400 & 47 & Green & 13 & 6400 & 45 & Red \\
6 & 2900 & 75 & Red & 14 & 6900 & 17 & Green \\
7 & 3400 & 10 & Red & 15 & 7400 & 5 & Red \\
8 & 3900 & 2 & Green & 16 & 7900 & 35 & Red \\
\hline
\end{tabular}


TABLE 4.

Related Parameters of IDM and VT-CPFM Model

\begin{tabular}{ccc|ccc|ccc}
\hline Symbol & Typical Value & Unit & Symbol & Typical Value & Unit & Symbol & Typical Value & Unit \\
\hline$T$ & 0.5 & $\mathrm{~s}$ & $v_{d}$ & 6.94 & $\mathrm{~m} / \mathrm{s}$ & $\delta$ & 4 & $\backslash$ \\
$a$ & 3 & $\mathrm{~m} / \mathrm{s}^{2}$ & $b$ & 3 & $\mathrm{~m} / \mathrm{s}^{2}$ & $s_{\min }$ & 4 & $\mathrm{~m}$ \\
$T_{s}$ & 1 & $\mathrm{~s}$ & $L$ & 12 & $\mathrm{~m}$ & $D_{d}$ & 300 & $\mathrm{~m}$ \\
$C_{d i s t}$ & 11 & $m$ & $g$ & 9.81 & $\mathrm{~m} / \mathrm{s}^{2}$ & $C_{d}$ & 0.3 & $\backslash$ \\
$C_{h}$ & 0.95 & $\backslash$ & $\alpha_{0}$ & $1.192 \times 10^{-3}$ & $\backslash$ & $\alpha_{1}$ & $1.059 \times 10^{-4}$ & $\backslash$ \\
$\alpha_{2}$ & $-1.738 \times 10^{-7}$ & $\backslash$ & $m_{b}$ & 17996.3 & $\mathrm{~kg}$ & $\eta_{d}$ & 0.95 & $\mathrm{~s}$ \\
$A_{f}$ & 6.824 & $\mathrm{~m}^{2}$ & $\rho$ & 1.2256 & $\mathrm{~kg} / \mathrm{m}^{3}$ & $C_{r 0}$ & 1.25 & $\backslash$ \\
$C_{r 1}$ & 0.0328 & $\backslash$ & $C_{r 2}$ & 4.575 & $\backslash$ & $m_{p}$ & 70 & $\mathrm{~kg}$ \\
\hline
\end{tabular}

To mitigate bus bunching and reduce the possibility of reaching the intersection during the red phase, the AASP algorithm is used to ensure that the bus can reach the next stop with the least delays. However, the proposed AASP algorithm needs a bus arrival schedule to plan its anticipated average speed. This paper takes both constant and adaptive time schedules into account. The constant time schedule (CTS) is given as $a_{j, i}^{\text {schedule }}=$ $a_{j-1, i}^{\text {schedle }}+t_{H c}$, where $t_{H c}$ is a constant. The adaptive time schedule (ATS) is given as $a_{j, i}^{\text {schedule }}=a_{j-1, i}+t_{H}$ where $t_{H}$ is an adjustable parameter.

The first bus time schedule given by Table 5 is used to calculate the arrival schedules of bus followers. The formula for ATS shows that the adaptive time schedule of bus $j$ can be updated only when bus $j-1$ has arrived at the target stop. However, this is difficult to realize, especially when two successive buses show a tendency toward bus bunching. To overcome this problem, all follower arrival schedules are updated by executing $a_{l l+1, i}^{\text {schedule }}+a_{l l, i}^{\text {schedule }}+t_{H}$ with $l l=j, j+1 \cdots, N$ immediately after updating the arrival schedule of bus $j$ by $a_{j, i}^{\text {schedule }}$ $=a_{j-1, i}+t_{H}$ in state 2. Meanwhile, $a_{j, i+1}^{\text {schedule }}=d_{j, i}+\left(\sum_{k=1}^{k l}\left(a_{k, i+1}-d_{k, i}\right) \cdot 2^{k}\right) /\left(\sum_{k=1}^{k l} 2^{k}\right)+\min \left(0.1 \cdot t_{H,} \max (0.8 \cdot\right.$ $\left.\left.\left[t_{H}-\left(d_{j, i}-d_{j-1, i}\right)\right],-0.1 \cdot t_{H}\right)\right)$ is used to re-update the arrival schedule of bus $j$ at stop $i+1$ when bus $j$ prepares to go ahead to intersection $i+1$, and bus $j-1$ has not yet departed from stop $i+1$, where $k l \leq j-1$ and $p_{k l} \geq p_{b, i+1}$. In the simulation, $\mathrm{HC}$ and AASP are combined to adjust headway regularity. The holding logic is that the bus can depart from the stop only if its dwell time is greater than the average headway between succeeding buses in the same line.

TABLE 5.

Arrival Schedules of the First Bus at Each Bus Stop

\begin{tabular}{lcccccccc}
\hline Bus Stop Number & $\mathbf{1}$ & $\mathbf{2}$ & $\mathbf{3}$ & $\mathbf{4}$ & $\mathbf{5}$ & $\mathbf{6}$ & $\mathbf{7}$ & $\mathbf{8}$ \\
\hline Position (km) & 0.5 & 1.0 & 1.5 & 2.0 & 2.5 & 3.0 & 3.5 & 4.0 \\
Arrival Schedule (s) & 102 & 295 & 392 & 554 & 692 & 862 & 988 & 1087 \\
\hline Bus Stop Number & $\mathbf{9}$ & $\mathbf{1 0}$ & $\mathbf{1 1}$ & $\mathbf{1 2}$ & $\mathbf{1 3}$ & $\mathbf{1 4}$ & $\mathbf{1 5}$ & $\mathbf{1 6}$ \\
\hline Position (km) & 4.5 & 5.0 & 5.5 & 6.0 & 6.5 & 7.0 & 7.5 & 8.0 \\
Arrival Schedule (s) & 1234 & 1333 & 1445 & 1583 & 1743 & 1797 & 1926 & 2054 \\
\hline
\end{tabular}


Passenger arrival rate is assumed to follow a uniform distribution with a mean of 180 passengers per hour $(\mathrm{pax} / \mathrm{h})$ for the case without special statement. Alighting rates at each stop are given as $0,0.1,0.3,0.5,0.5,0.5$, $0.6,0.8,0.8,0.8,0.8,0.8,0.8,0.8,0.8,0.8$, respectively. The boarding rate is set as 15 passengers per minute (pax/min), and the alighting rate is set as two seconds per passenger ( $2 \mathrm{~s} / \mathrm{pax})$. Bus capacity is set as 60 passengers. The statistical least service time is set as $0.7 t_{H}$ for human-driven bus and $t_{H}$ for connected bus. The constant $\kappa, \varepsilon_{s}$ and $\varepsilon_{V}$ are set as $0.5,10^{-4}$, and $10^{-4}$, respectively.

\section{Simulation for Human-Driven Bus Propagation Model}

Figure 6 shows the spatial-temporal responses of the human-driven bus propagation model considering signal control and passenger swapping behavior under a different passenger swapping rate (psr), where $v_{d}=25 \mathrm{~km} / \mathrm{h}$, demand $=180 \mathrm{pax} / \mathrm{h}$, service frequency $=10.5 \mathrm{bus} / \mathrm{h}$, and cycle length $=120 \mathrm{~s}$. As demonstrated by Figure 6 , bus motion obeys signal control very well and the bus can stop at the corresponding bay according to arrival sequences.

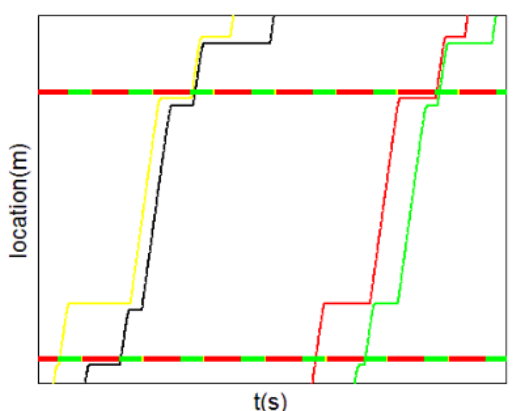

(a) $p s r=0$

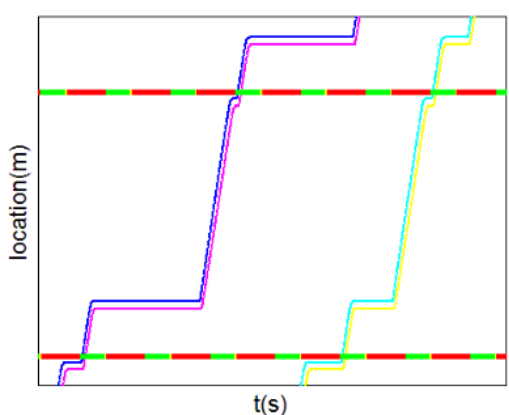

(b) $p s r=1$

\section{FIGURE 6.}

Responses of human-driven bus propagation model under different passenger swapping rates

To mitigate bus bunching, psr is used to manage passenger swapping behaviors. According to Figure 6(a), the bunching front bus will depart prior to the rear one with a longer time when $p s r=0.0$, since the bunching front bus only needs to finish passenger alighting, while the rear one needs to load almost all remaining passengers, which in turn recovers the headway regularity. However, according to Figure 6(b), the bunching front bus has to load all remaining passengers and the rear one leaves the stop immediately after the bunching front leaves when $p s r=1.0$, which in turn has no positive effect on mitigating bus bunching. Since bunching front is probably to load more passengers than the rear one at the next stop, the bunching front and rear buses may bunch again at subsequent stops. In summary, designing a passenger service system with smaller passenger swapping rates can be a countermeasure to mitigate bus bunching, but it is not enough to fully eliminate bunching.

Figure 7 shows the spatial-temporal responses of the human-driven bus propagation model considering signal control and passenger swapping behavior under different passenger demand, where $v_{d}=25 \mathrm{~km} / \mathrm{h}, p s r=0.0$, service frequency $=10.5$ bus $/ \mathrm{h}$, and cycle length $=120 \mathrm{~s}$. 


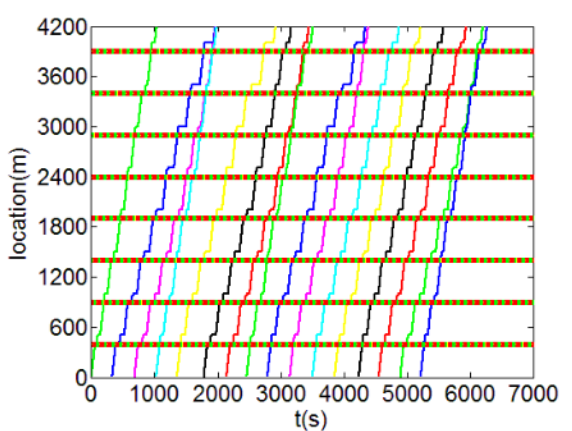

(a.1) demand = $135 \mathrm{pax} / \mathrm{h}$

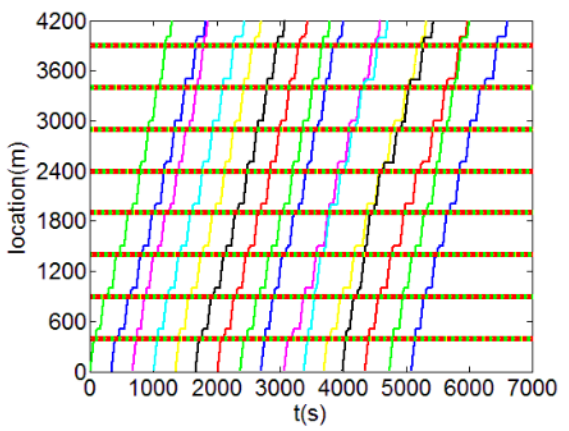

(b.1) demand = $180 \mathrm{pax} / \mathrm{h}$

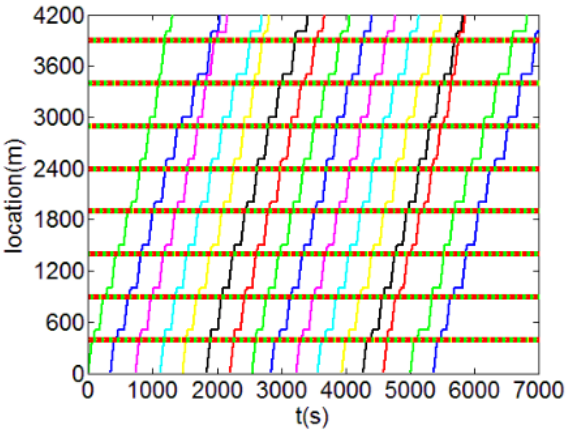

(c.1) demand $=225 \mathrm{pax} / \mathrm{h}$

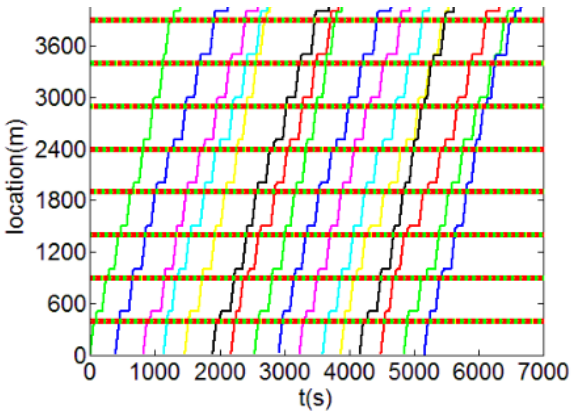

(d.1) demand $=270 \mathrm{pax} / \mathrm{h}$

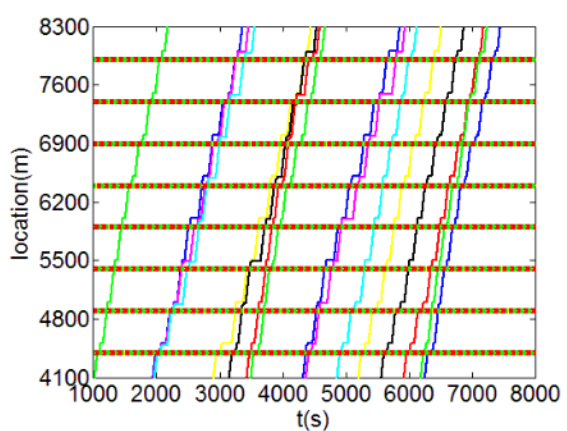

(a.2) demand = $135 \mathrm{pax} / \mathrm{h}$

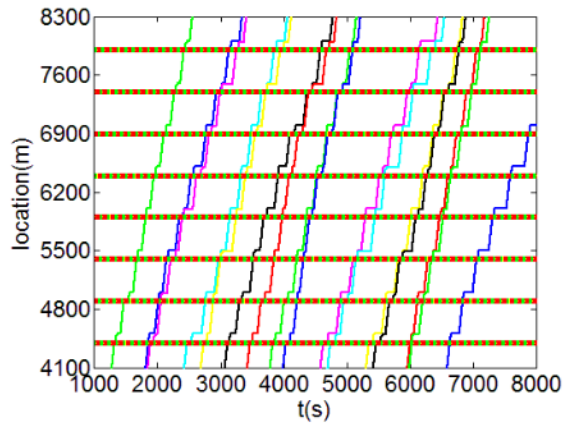

(b.2) demand = $180 \mathrm{pax} / \mathrm{h}$

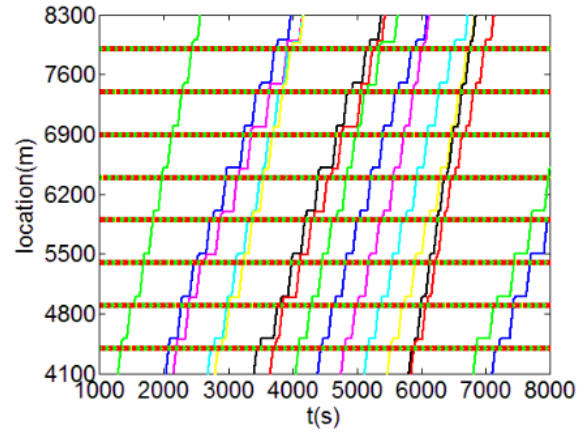

(c.2) demand = $225 \mathrm{pax} / \mathrm{h}$

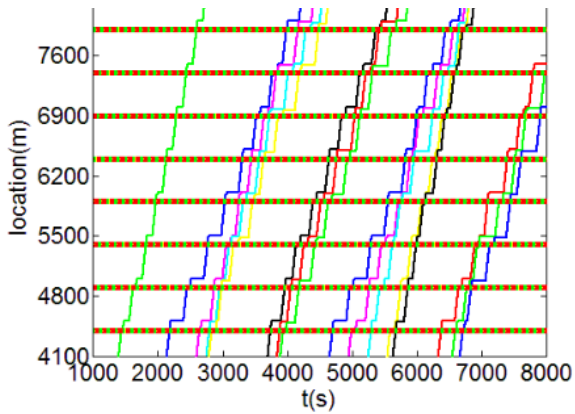

(d.2) demand = $270 \mathrm{pax} / \mathrm{h}$

\section{FIGURE 7.}

Responses of human-driven bus propagation model under different passenger demand 
Figure 7 also reveals some phenomena: less bus bunching occurs at the first eight bus stops than the last eight bus stops; the bus motion at the first eight bus stops is almost the same, but quite different from one another at the last eight bus stops; and bus bunching becomes more severe as stop numbers increase. These phenomena can be explained as all buses depart from the bus terminal with uniform headways and arrive at the first eight stops with less cumulative disturbances caused by passenger demand variations, unexpected signals, and initial differences. When bus headway deviates from its nominal value, passenger demand will act as positive feedback to the bus propagation model in subsequent stops, which in turn reduces the service regularity and causes bunching. When bunching occurs, the headway varies greatly since the bunching front has a greater headway than other bunching members. Moreover, the more buses bunching together, the greater the bunching front headway becomes. As is shown by Figure 7 (a.2-d.2), the greater the passenger demand, the more severe the bunching that occurs according to bunching number and travel delays.

Table 6 shows some performance metrics of the human-driven bus propagation model considering signal control and passenger swapping behavior with demand $=180 \mathrm{pax} / \mathrm{h}, v_{d}=25 \mathrm{~km} / \mathrm{h}$, service frequency $=10.5$ bus $/ \mathrm{h}$, and cycle length $=120 \mathrm{~s}$. According to Table 6, buses tend to bunch no matter what the passenger swapping rate is, and the mean value of headway, mean value of waiting time for a green signal, and average load factor seem to be unrelated to the variation of psr. When psr is less than 0.5 , both travel time per $\mathrm{km}$ and mean value of dwell time do not change substantially. However, these two metrics vary and grow with the increment of $p s r$ when $p s r \geq 0.5$. Travel time per $\mathrm{km}$ and mean value of dwell time under $p s r \geq 0.5$ are all greater than under $p s r<0.5$. When $p s r$ is less than 0.5 , the bunching rate, standard deviation of headway, and passenger waiting time show no relation to $p s r$, but these three metrics decline first and then grow with the increment of $p s r$ when $p s r \geq 0.5$. Additionally, the fuel efficiency grows with the increment of $p s r$ except when $p s r=0.2$.

\section{TABLE 6.}

Impact of Passenger Swapping Rate on Bus Operational Performance

\begin{tabular}{cccccccccc}
\hline psr & $\begin{array}{c}\text { BR } \\
\text { (\%) }\end{array}$ & $\begin{array}{c}\text { M-DT } \\
\text { (s) }\end{array}$ & $\begin{array}{c}\text { M-WTGL } \\
\text { (s) }\end{array}$ & $\begin{array}{c}\text { M-TH } \\
\text { (s) }\end{array}$ & $\begin{array}{c}\text { STD-TH } \\
\text { (s) }\end{array}$ & $\begin{array}{c}\text { TTPK } \\
\text { (s/km) }\end{array}$ & $\begin{array}{c}\text { PWT } \\
\text { (s) }\end{array}$ & $\begin{array}{c}\text { ALF } \\
\text { (\%) }\end{array}$ & $\begin{array}{c}\text { FE } \\
(\text { L/100 km) }\end{array}$ \\
\hline 0.0 & 31 & 72 & 25 & 351 & 203 & 336.8 & 246 & 45 & 63.6 \\
0.1 & 36 & 74 & 25 & 365 & 238 & 340.1 & 274 & 46 & 64.1 \\
0.2 & 27 & 71 & 24 & 350 & 196 & 333.4 & 236 & 45 & 63.3 \\
0.3 & 41 & 73 & 27 & 360 & 273 & 341.8 & 294 & 46 & 64.6 \\
0.4 & 42 & 73 & 26 & 359 & 262 & 340.1 & 282 & 46 & 64.3 \\
0.5 & 43 & 73 & 25 & 365 & 298 & 340.3 & 309 & 46 & 64.8 \\
0.6 & 34 & 74 & 27 & 353 & 243 & 345.0 & 261 & 45 & 65.1 \\
0.7 & 28 & 78 & 24 & 364 & 236 & 345.9 & 261 & 46 & 65.1 \\
0.8 & 32 & 79 & 24 & 352 & 237 & 347.7 & 257 & 45 & 65.2 \\
0.9 & 43 & 85 & 25 & 357 & 305 & 362.4 & 311 & 46 & 67.3 \\
1.0 & 42 & 88 & 25 & 356 & 310 & 368.0 & 316 & 46 & 67.9 \\
\hline
\end{tabular}

By comparing all metrics, we find that decreasing the passenger swapping rate produces more benefits than other settings. Hence in practice, encouraging passengers to take the rear bus when bunching occurs is a good countermeasure to mitigate bus bunching on the condition that overtaking is forbidden. Although the passenger swapping rate can be changed to improve operational performance when bunching occurs, this countermeasure does not completely remove bus bunching. 
To reveal the causes of bus bunching, the impacts of some related factors on bus performance are analyzed, including service frequency, route length, and signal cycle length.

\section{Impact Analysis of Service Frequency on Human-Driven Bus Operation}

Table 7 shows the impact of service frequency on human-driven bus performance with passenger demand $(P D)$, cycle length $=120 \mathrm{~s}, v_{d}=25 \mathrm{~km} / \mathrm{h}, p s r=0.0$, and stop number $=16$. In the following three cases, the mean value of dwell time, travel time per $\mathrm{km}$, average load factor, and fuel efficiency all increase with the increment of demand and service frequency, while waiting time for a green signal increases only with the increment of service frequency when demand $\geq 180 \mathrm{pax} / \mathrm{h}$. Additionally, it is noteworthy that the departure headway at the bus terminal is less than the mean value of headway, due to the effect of signal control.

TABLE 7.

Impact of Service Frequency on Human-Driven Bus Performance

\begin{tabular}{ccccccccccc}
\hline $\begin{array}{c}\text { Service } \\
\begin{array}{c}\text { Frequency } \\
\text { (bus/h) }\end{array}\end{array}$ & $\begin{array}{c}\text { PD } \\
\text { (pax/h) }\end{array}$ & $\begin{array}{c}\text { BR } \\
\text { (\%) }\end{array}$ & $\begin{array}{c}\text { M-DT } \\
\text { (s) }\end{array}$ & $\begin{array}{c}\text { M-WTGL } \\
\text { (s) }\end{array}$ & $\begin{array}{c}\text { M-TH } \\
\text { (s) }\end{array}$ & $\begin{array}{c}\text { STD-TH } \\
\text { (s) }\end{array}$ & $\begin{array}{c}\text { TTPK } \\
\text { (s/km) }\end{array}$ & $\begin{array}{c}\text { PWT } \\
\text { (s) }\end{array}$ & $\begin{array}{c}\text { ALF } \\
\text { (\%) }\end{array}$ & $\begin{array}{c}\text { FE } \\
\text { (L/100 } \\
\text { km) }\end{array}$ \\
\hline 15.75 & 270 & 56 & 85 & 27 & 266 & 278 & 365.4 & 222 & 50 & 67.4 \\
10.5 & 270 & 51 & 113 & 24 & 372 & 303 & 410.1 & 393 & 69 & 72.9 \\
7.875 & 270 & 24 & 140 & 23 & 504 & 263 & 459.3 & 719 & 87 & 79.0 \\
\hline 15.75 & 225 & 51 & 69 & 31 & 258 & 272 & 342.6 & 200 & 41 & 64.7 \\
10.5 & 225 & 52 & 95 & 24 & 366 & 297 & 376.2 & 341 & 58 & 68.6 \\
7.875 & 225 & 38 & 122 & 21 & 487 & 330 & 421.4 & 418 & 76 & 74.1 \\
\hline 15.75 & 180 & 62 & 53 & 35 & 246 & 262 & 320.5 & 193 & 31 & 62.1 \\
10.5 & 180 & 31 & 72 & 25 & 351 & 203 & 336.8 & 246 & 45 & 63.6 \\
7.875 & 180 & 50 & 100 & 24 & 485 & 352 & 386.9 & 311 & 61 & 70.0 \\
\hline 15.75 & 135 & 64 & 39 & 29 & 241 & 182 & 281.5 & 153 & 23 & 56.7 \\
10.5 & 135 & 49 & 60 & 31 & 376 & 302 & 325.4 & 328 & 36 & 62.5 \\
7.875 & 135 & 10 & 71 & 23 & 471 & 183 & 329.6 & 258 & 45 & 62.7 \\
\hline
\end{tabular}

Case 1: When service frequency is high, bus bunching is likely to occur for all demand levels, since the bunching rates are greater than $50 \%$ and the ratio of headway mean value to its standard deviation is also greater than $75 \%$. Both headway mean value and passenger waiting time increase monotonically with the increment of demand in Case 1.

Case 2: When service frequency is medium, bus bunching is likely to occur for all demands except $180 \mathrm{pax} / \mathrm{h}$. In Case 2, the bunching rate declines and then increases with the increment of demand. Passenger waiting time is not fully dependent on demand since the minimum value of passenger waiting time occurs at demand $=180$ $\mathrm{pax} / \mathrm{h}$ instead of $135 \mathrm{pax} / \mathrm{h}$.

Case 3: When service frequency is low, bus bunching tends to be mitigated for all demands except $180 \mathrm{pax} / \mathrm{h}$. In Case 3, passenger waiting time increases monotonically with the increment of demand and increases sharply when the average load factor exceeds about $80 \%$. The bunching rate grows and then declines with the increment of demand, indicating that bus bunching can be mitigated when the average load factor or demand is quite high or quite small. 
In summary, bus bunching is prone to occur at high service frequency. This is because small headway in a high service frequency condition is more sensitive to disturbances than large headway in a low service frequency condition.

\section{Impact Analysis of Route Length on Human-Driven Bus Operation}

Table 8 shows the impact of route length on human-driven bus performance with cycle length $=120 \mathrm{~s}$, service frequency $=10.5$ bus $/ \mathrm{h}, v_{d}=25 \mathrm{~km} / \mathrm{h}$, and $p s r=0.0$. Here stop number is used to represent route length. According to Table 8, the bunching rate, mean value of dwell time, mean value and standard deviation of headway, travel time per $\mathrm{km}$, and passenger waiting time all increase with the increment of stop number, while the average load factor decreases with the increment of demand and stop number. In addition, both mean value of waiting time for a green signal and fuel efficiency are irrelevant to stop number. In summary, bus bunching has a higher probability of occurring on a longer route than on a shorter route.

\section{TABLE 8.}

Impact of Route Length on Human-Driven Bus Operational Performance

\begin{tabular}{ccccccccccc}
\hline $\begin{array}{c}\text { Stop } \\
\text { Number }\end{array}$ & $\begin{array}{c}\text { PD } \\
\text { (pax/h) }\end{array}$ & $\begin{array}{c}\text { BR } \\
(\%)\end{array}$ & $\begin{array}{c}\text { M-DT } \\
\text { (s) }\end{array}$ & $\begin{array}{c}\text { M-WTGL } \\
\text { (s) }\end{array}$ & $\begin{array}{c}\text { M-TH } \\
\text { (s) }\end{array}$ & $\begin{array}{c}\text { STD-TH } \\
(\mathbf{s})\end{array}$ & $\begin{array}{c}\text { TTPK } \\
(\mathbf{s} / \mathbf{k m})\end{array}$ & $\begin{array}{c}\text { PWT } \\
(\mathbf{s})\end{array}$ & $\begin{array}{c}\text { ALF } \\
(\%)\end{array}$ & $\begin{array}{c}\text { FE } \\
(\mathbf{L} / \mathbf{1 0 0} \\
\mathbf{k m})\end{array}$ \\
\hline 8 & 270 & 26 & 107 & 22 & 356 & 161 & 388.9 & 278 & 80 & 70.7 \\
12 & 270 & 41 & 110 & 22 & 363 & 236 & 409.5 & 323 & 73 & 73.0 \\
16 & 270 & 51 & 113 & 24 & 372 & 303 & 410.1 & 393 & 69 & 72.9 \\
\hline 8 & 225 & 25 & 89 & 21 & 353 & 151 & 360.4 & 217 & 67 & 67.4 \\
12 & 225 & 42 & 93 & 22 & 359 & 224 & 375.1 & 271 & 61 & 68.8 \\
16 & 225 & 52 & 95 & 24 & 366 & 297 & 376.2 & 341 & 58 & 68.6 \\
\hline 8 & 180 & 4 & 69 & 26 & 346 & 88 & 329.3 & 184 & 52 & 63.4 \\
12 & 180 & 17 & 70 & 25 & 348 & 141 & 336.9 & 205 & 47 & 63.9 \\
16 & 180 & 31 & 72 & 25 & 351 & 203 & 336.8 & 246 & 45 & 63.6 \\
\hline 8 & 135 & 33 & 56 & 33 & 365 & 197 & 323.6 & 238 & 42 & 62.9 \\
12 & 135 & 43 & 59 & 31 & 371 & 257 & 327.4 & 286 & 38 & 63.0 \\
16 & 135 & 49 & 60 & 31 & 376 & 302 & 325.4 & 328 & 36 & 62.5 \\
\hline
\end{tabular}

\section{Impact Analysis of Signal Cycle Time on Human-Driven Bus Operation}

Table 9 shows the impact of signal cycle length on human-driven bus operational performance with service frequency $=10.5$ bus $/ \mathrm{h}$, stop number $=16, v_{d}=25 \mathrm{~km} / \mathrm{h}$, and $p s r=0.0$. According to Table 9, the following conclusions can be drawn: the mean value and standard deviation of waiting time for a green signal, and travel time per km, decline with the decrement of signal cycle length; fuel efficiency almost decreases with the decrement of signal cycle length except when demand $=135 \mathrm{pax} / \mathrm{h}$; and bunching rate, mean value of dwell time, mean value of headway, passenger waiting time, and average load factor do not show a substantial linear relationship with signal cycle length. In summary, as for fixed-time signals, bus operation can benefit from proper signal cycle length rather than shorter or longer cycle length. 
TABLE 9.

Impact of Signal Cycle Time on Human-Driven Bus Operational Performance

\begin{tabular}{cccccccccc}
\hline $\begin{array}{c}\text { Cycle Time } \\
\text { (s) }\end{array}$ & $\begin{array}{c}\text { PD } \\
\text { (pax/h) }\end{array}$ & $\begin{array}{c}\text { BR } \\
\text { (\%) }\end{array}$ & $\begin{array}{c}\text { M-DT } \\
\text { (s) }\end{array}$ & $\begin{array}{c}\text { M-/STD-WTGL } \\
\text { (s) }\end{array}$ & $\begin{array}{c}\text { M-/STD-TH } \\
\text { (s) }\end{array}$ & $\begin{array}{c}\text { TTPK } \\
(\mathbf{s} / \mathbf{k m})\end{array}$ & $\begin{array}{c}\text { PWT } \\
\text { (s) }\end{array}$ & $\begin{array}{c}\text { ALF } \\
\text { (\%) }\end{array}$ & $\begin{array}{c}\text { FE } \\
\text { (L/100 km) }\end{array}$ \\
\hline 180 & 270 & 40 & 122 & $57 / 42$ & $410 / 326$ & 491.1 & 492 & 75 & 83.1 \\
120 & 270 & 41 & 110 & $22 / 26$ & $363 / 236$ & 409.5 & 431 & 73 & 73.0 \\
60 & 270 & 48 & 111 & $11 / 13$ & $370 / 330$ & 383.2 & 421 & 69 & 69.7 \\
\hline 180 & 225 & 68 & 103 & $41 / 42$ & $396 / 375$ & 426.4 & 452 & 61 & 74.6 \\
120 & 225 & 42 & 93 & $22 / 25$ & $359 / 224$ & 375.1 & 361 & 61 & 68.8 \\
60 & 225 & 63 & 97 & $12 / 14$ & $370 / 329$ & 358.7 & 374 & 59 & 66.6 \\
\hline 180 & 180 & 59 & 79 & $38 / 41$ & $367 / 324$ & 374.7 & 356 & 47 & 67.9 \\
120 & 180 & 17 & 70 & $25 / 25$ & $348 / 141$ & 336.9 & 273 & 47 & 63.9 \\
60 & 180 & 58 & 79 & $12 / 13$ & $385 / 300$ & 325.6 & 333 & 48 & 62.6 \\
\hline 180 & 135 & 74 & 61 & $33 / 41$ & $354 / 364$ & 330.0 & 393 & 34 & 62.0 \\
120 & 135 & 43 & 59 & $31 / 26$ & $371 / 257$ & 327.4 & 381 & 38 & 63.0 \\
60 & 135 & 44 & 56 & $13 / 13$ & $359 / 276$ & 282.3 & 299 & 34 & 57.0 \\
\hline
\end{tabular}

\section{Simulation for Connected Bus Propagation Model}

Figure 8 shows the spatial-temporal responses of the connected bus propagation process under adaptive time schedule (ATS) based AASP and HC with service frequency $=10.5$ bus $/ \mathrm{h}$, cycle length $=120 \mathrm{~s}$, and red ratio $=$ 0.6 . As shown by Figure 8, all buses can keep regular headways and bunching is removed thoroughly for demand ranging from 135 to $270 \mathrm{pax} / \mathrm{h}$. This is because ATS based AASP and HC limits the disturbances propagating from the bus leader to followers, caused by passenger demand variation, initial delays, and signal control. Hence the followers can easily maintain a regular headway. With the increment of demand, the mean values of travel time, dwell time, and headway increase. As Figure 8 illustrates, a connected bus can maintain a regular headway and reduce travel time at the same time. 


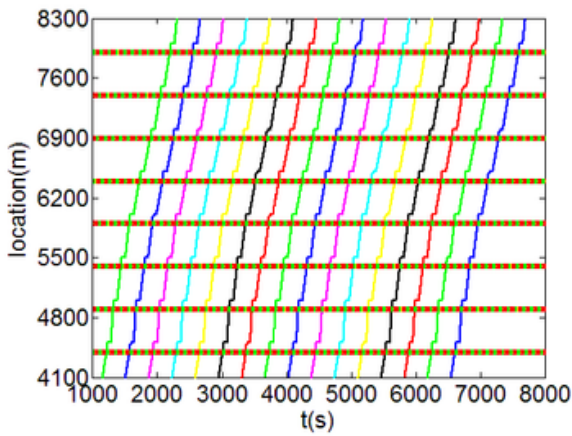

(a) demand $=135 \mathrm{pax} / \mathrm{h}$

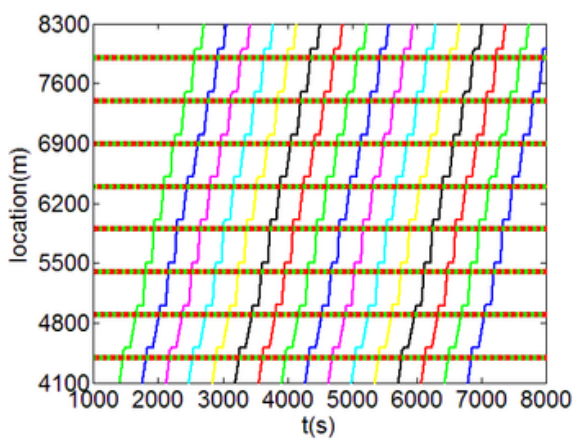

(c) demand $=225 \mathrm{pax} / \mathrm{h}$

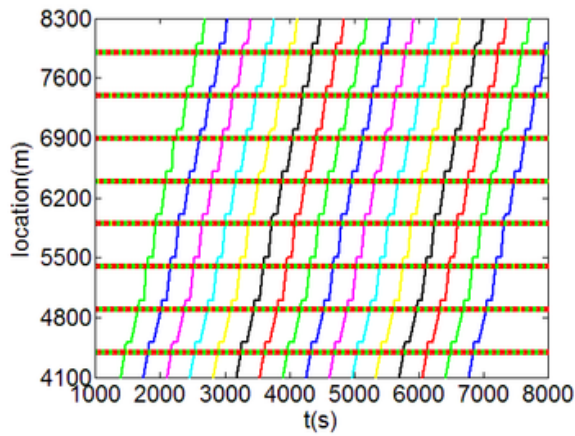

(b) demand $=180 \mathrm{pax} / \mathrm{h}$

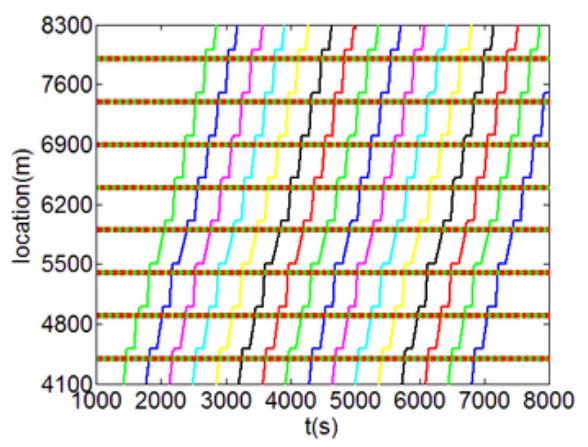

(d) demand $=270 \mathrm{pax} / \mathrm{h}$

\section{FIGURE 8.}

Responses of connected bus propagation model under ATS based AASP and HC

Table 10 shows the impact of service frequency on the connected bus propagation model with AASP and $\mathrm{HC}$, where cycle length $=120 \mathrm{~s}$ and red ratio $=0.6$. The connected bus with ATS based AASP and HC can greatly improve bus line performance: bus bunching is removed successfully for all mentioned settings of passenger demand and service frequency; compared with the human-driven bus (Table 8), the mean value of dwell time is reduced or kept invariant, while mean value of waiting time for a green signal, standard deviation of headway, travel time per $\mathrm{km}$, and passenger waiting time are all reduced significantly; and the average load factor and fuel efficiency do not show any significant improvements. In summary, the connected bus with ATS based AASP and HC is robust to mitigate bunching against passenger demand variations and different service frequencies. 
TABLE 10.

Impact of Service Frequency on Connected Bus Propagation Model

\begin{tabular}{ccccccccccc}
\hline $\begin{array}{c}\text { SF } \\
\text { (bus/h) }\end{array}$ & $\begin{array}{c}\text { PD } \\
\text { (pax/h) }\end{array}$ & $\begin{array}{c}\text { BR } \\
\text { (\%) }\end{array}$ & $\begin{array}{c}\text { M-DT } \\
\text { (s) }\end{array}$ & $\begin{array}{c}\text { M-WTGL } \\
\text { (s) }\end{array}$ & $\begin{array}{c}\text { M-TH } \\
\text { (s) }\end{array}$ & $\begin{array}{c}\text { STD-TH } \\
\text { (s) }\end{array}$ & $\begin{array}{c}\text { TTPK } \\
\text { (s/km) }\end{array}$ & $\begin{array}{c}\text { PWT } \\
\text { (s) }\end{array}$ & $\begin{array}{c}\text { ALF } \\
\text { (\%) }\end{array}$ & $\begin{array}{c}\text { FE } \\
(\mathbf{L} / \mathbf{1 0 0} \mathbf{~ k m ) ~}\end{array}$ \\
\hline 15.75 & 270 & 0 & 72 & 1 & 238 & 12 & 318.8 & 120 & 46 & 63.9 \\
10.5 & 270 & 0 & 107 & 2 & 357 & 20 & 352.5 & 185 & 68 & 70.8 \\
7.875 & 270 & 0 & 133 & 1 & 479 & 33 & 442.3 & 516 & 84 & 78.7 \\
\hline 15.75 & 225 & 0 & 59 & 1 & 238 & 11 & 314.0 & 119 & 38 & 67.1 \\
10.5 & 225 & 0 & 90 & 2 & 360 & 7 & 321.6 & 180 & 58 & 64.3 \\
7.875 & 225 & 0 & 117 & 1 & 477 & 34 & 374.6 & 308 & 75 & 70.6 \\
\hline 15.75 & 180 & 0 & 48 & 1 & 239 & 7 & 271.4 & 120 & 31 & 57.4 \\
10.5 & 180 & 0 & 72 & 2 & 358 & 16 & 324.1 & 180 & 46 & 67.2 \\
7.875 & 180 & 0 & 95 & 1 & 476 & 27 & 343.6 & 239 & 61 & 72.0 \\
\hline 15.75 & 135 & 0 & 36 & 1 & 239 & 9 & 260.1 & 120 & 23 & 57.5 \\
10.5 & 135 & 0 & 53 & 2 & 358 & 19 & 290.7 & 179 & 34 & 63.9 \\
7.875 & 135 & 0 & 71 & 1 & 477 & 15 & 322.8 & 239 & 46 & 69.8 \\
\hline
\end{tabular}

Table 11 shows the impact of cycle length on the connected bus propagation model, where service frequency = 10.5 bus/h. The smaller cycle length needed to extend to more cycles (Table 2) adds great complexity to AASP but will not bring great benefits, thus only ATS based HC is used to mitigate bus bunching for cycle length = 60 s. As Table 11 illustrates, a connected bus can essentially remove bus bunching for all levels of passenger demand and cycle length.

TABLE 11.

Impact of Cycle Time on Connected Bus Propagation Model

\begin{tabular}{cccccccccccc}
\hline $\begin{array}{c}\text { CT } \\
\text { (s) }\end{array}$ & $\begin{array}{c}\text { PD } \\
(\mathbf{p a x} / \mathbf{h})\end{array}$ & $\begin{array}{c}\text { BR } \\
(\%)\end{array}$ & $\begin{array}{c}\text { M-DT } \\
\text { (s) }\end{array}$ & $\begin{array}{c}\text { M-WTGL } \\
\text { (s) }\end{array}$ & $\begin{array}{c}\text { M-TH } \\
\text { (s) }\end{array}$ & $\begin{array}{c}\text { STD-TH } \\
(\mathbf{s})\end{array}$ & $\begin{array}{c}\text { TTPK } \\
(\mathbf{s} / \mathbf{k m})\end{array}$ & $\begin{array}{c}\text { PWT } \\
(\mathbf{s})\end{array}$ & $\begin{array}{c}\text { ALF } \\
(\%)\end{array}$ & $\begin{array}{c}\text { FE } \\
(\mathbf{L} / \mathbf{1 0 0} \mathbf{k m})\end{array}$ & Method \\
\hline 180 & 270 & 7 & 113 & 4 & 381 & 91 & 426.0 & 232 & 72 & 76.3 & HC+AASP \\
120 & 270 & 0 & 107 & 2 & 357 & 20 & 352.5 & 185 & 68 & 70.8 & HC+AASP \\
60 & 270 & 0 & 119 & 16 & 351 & 11 & 398.9 & 179 & 67 & 75.2 & HC \\
\hline 180 & 225 & 0 & 90 & 3 & 358 & 15 & 372.9 & 179 & 58 & 68.0 & HC+AASP \\
120 & 225 & 0 & 90 & 2 & 360 & 7 & 321.6 & 180 & 58 & 64.3 & HC+AASP \\
60 & 225 & 3 & 131 & 13 & 361 & 76 & 417.1 & 190 & 57 & 76.7 & HC \\
\hline 180 & 180 & 0 & 71 & 2 & 356 & 19 & 346.2 & 179 & 45 & 68.1 & HC+AASP \\
120 & 180 & 0 & 72 & 2 & 358 & 16 & 324.1 & 180 & 46 & 67.2 & HC+AASP \\
60 & 180 & 0 & 85 & 12 & 351 & 12 & 327.0 & 176 & 45 & 66.3 & HC \\
\hline 180 & 135 & 0 & 54 & 3 & 357 & 16 & 291.1 & 179 & 34 & 62.0 & HC+AASP \\
120 & 135 & 0 & 53 & 2 & 358 & 19 & 290.7 & 179 & 34 & 63.9 & HC+AASP \\
60 & 135 & 0 & 60 & 14 & 365 & 27 & 283.0 & 183 & 35 & 62.4 & HC \\
\hline
\end{tabular}


Compared with the human-driven bus (Table 9), the connected bus can reduce both standard deviation of headway and passenger waiting time significantly. The connected bus with AASP and HC can reduce the mean value of dwell time and mean value of waiting time for a green signal, while the connected bus with $\mathrm{HC}$ increases the mean value of dwell time and does not affect the mean value of waiting time for a green signal. The connected bus propagation model can be nearly free of bus bunching under different demand and cycle length values, which can generate bus trajectories and holding commands for a connected bus en route and help the bus line mitigate bus bunching.

\section{Conclusions}

To improve bus operations and performance, an extended bus propagation model based on a finite state machine was established accounting for signal and anti-bunching control. The model extended the twobunching bus propagation model to a generic form and extended the time-based bus propagation model to a spatial-temporal based bus propagation model, suitable for describing the dynamic motion affected by signal control and providing for anti-bunching by speed adjustment. This model can be extended to consider different passenger arrival distributions. To mitigate bus bunching, an AASP algorithm was designed to ensure that a connected bus could reach the next stop with the least delay and arrive at the next intersection stop bar during the green phase. Finally, MPC was used in an AASP algorithm to generate a smooth speed profile to follow anticipated average speed commands.

According to the simulation results, the following conclusions may be drawn:

1. The finite state machine can help ensure that the proposed human-driven bus propagation model considers signal control and passenger swapping behavior, and that all buses obey signal control rules.

2. A passenger service system can be used to automatically divide waiting passengers into groups to improve bunching bus performance, such as the mean value of waiting time for a green signal, travel time per $\mathrm{km}$, passenger waiting time, and fuel efficiency.

3. A connected bus with ATS based AASP and HC can essentially remove bus bunching and shows robustness against passenger demand, service frequency, and signal cycle length.

Bus bunching is a complex problem in practice that can be affected by many factors, such as congestion, V2X communication delays, sampling resolution of signal phasing and timing, and boarding demand from special groups. Passenger arrivals may not follow a uniform distribution when passengers use bus arrival predicting apps such as Mycitybus and Google Maps. The authors will be conducting further research on bus propagation modeling and analyzing the relative problems outlined in this paper.

\section{Acknowledgements}

The authors wish to express their gratitude for support from the National Natural Science Foundation of China (51775053, 71871028), National Key Research and Development Program of China (2019YFB1600500), Natural Science Basic Research plan in Shaanxi Province of China (2019JQ-691), and the Fundamental Research Funds for the Central Universities, China (300102220107). 


\section{References}

Altan, O. D., G. Wu, M. J. Barth, K. Boriboonsomsin, and J. A. Stark. 2017. "GlidePath: Eco-Friendly Automated Approach and Departure at Signalized Intersections." IEEE Transactions on Intelligent Vehicles 2 (4): 266-77. doi:10.1109/TIV.2017.2767289.

Asadi, B., and A. Vahidi. 2011. "Predictive Cruise Control: Utilizing Upcoming Traffic Signal Information for Improving Fuel Economy and Reducing Trip Time." IEEE Transactions on Control Systems Technology 19 (3): 707-14. doi:10.1109/TCST.2010.2047860.

Aziz, H. M. A., and S. V. Ukkusuri. 2016. "Network Traffic Control in Cyber-Transportation Systems Accounting for User-Level Fairness.” Journal of Intelligent Transportation Systems 20 (1): 4-16.

Cevallos, F., X. Wang, Z. Chen, and A. Gan. 2011. "Using AVL Data to Improve Transit On-Time Performance." Journal of Public Transportation 14 (3): 21-40. doi:10.5038/2375-0901.14.3.2.

Chien, S. I., Y. Ding, and C. Wei. 2002. "Dynamic Bus Arrival Time Prediction with Artificial Neural Networks." Journal of Transportation Engineering 128 (5): 429-38. doi:10.1061/(ASCE)0733-947X(2002)128:5(429).

Chow, A. H. F., and S. Li. 2017. "Modelling and Managing Bus Service Regularity with Influence of Prevailing Traffic." Transportmetrica B: Transport Dynamics 7 (1): 82-106. doi: 10.1080/21680566.2017.1353450.

Daganzo, C. F., and J. Pilachowski. 2011. "Reducing Bunching with Bus-to-Bus Cooperation." Transportation Research Part B: Methodological 45 (1): 267-77. doi:10.1016/j.trb.2010.06.005.

Eberlein, X. J., N. H. M. Wilson, and D. Bernstein. 2001. "The Holding Problem with Real-Time Information Available." Transportation Science 35 (1): 1-18. doi:10.1287/trsc.35.1.1.10143.

Fonzone, A., J.-D. Schmöcker, and R. Liu. 2015. "A Model of Bus Bunching under Reliability-based Passenger Arrival Patterns." Transportation Research Part C: Emerging Technologies 59: 164-82. doi:10.1016/j. trc.2015.05.020.

Han, J., A. Sciarretta, L. L. Ojeda, G. de Nunzio, and L. Thibault. 2018. "Safe- and Eco-Driving Control for Connected and Automated Electric Vehicles Using Analytical State-Constrained Optimal Solution." IEEE Transactions on Intelligent Vehicles 3 (2): 163-72. doi:10.1109/TIV.2018.2804162.

Huang, K., X. Yang, Y. Lu, C. C. Mi, and P. Kondlapudi. 2018. “Ecological Driving System for Connected/ Automated Vehicles Using a Two-Stage Control Hierarchy." IEEE Transactions on Intelligent Transportation Systems 19 (7): 2373-84.

Kesting, A., M. Treiber, M. Schönhof, and D. Helbing. 2008. "Adaptive Cruise Control Design for Active Congestion Avoidance." Transportation Research Part C: Emerging Technologies 16 (6): 668-83. doi:10.1016/j. trc.2007.12.004.

Li, S. E., S. Xu, X. Huang, B. Cheng, and H. Peng. 2015. "Eco-Departure of Connected Vehicles with V2X Communication at Signalized Intersections." IEEE Transactions on Vehicular Technology 64 (12): 5439-49. 
Li, S., R. Liu, L. Yang, and Z. Gao. 2019. "Robust Dynamic Bus Controls Considering Delay Disturbances and Passenger Demand Uncertainty." Transportation Research Part B: Methodological 123: 88-109. doi:10.1016/j. trb.2019.03.019.

Newell, G. F., and R. B. Potts. 1964. "Maintaining a Bus Schedule." Paper No. 170 in Proceedings of the Second Conference of the Australian Road Research Board 2 (1): 388-93.

Schmöcker, J.-D., W. Sun, A. Fonzone, and R. Liu. 2016. "Bus Bunching along a Corridor Served by Two Lines." Transportation Research Part B: Methodological 93 (Part A): 300-17. doi:10.1016/j.trb.2016.07.005.

Wang, C., Z. Ye, Y. Wang, Y. Xu, and W. Wang. 2016. “Modeling Bus Dwell Time and Time Lost Serving Stop in China." Journal of Public Transportation 19 (3): 55-77. doi:10.5038/2375-0901.19.3.4.

Wang, J., and H. A. Rakha. 2016. "Fuel Consumption Model for Conventional Diesel Buses." Applied Energy 170: 394-402. doi:10.1016/j.apenergy.2016.02.124.

Wu, W., R. Liu, and W. Jin. 2017. "Modelling Bus Bunching and Holding Control with Vehicle Overtaking and Distributed Passenger Boarding Behavior." Transportation Research Part B: Methodological 104: 175-97. doi:10.1016/J.TRB.2017.06.019.

Xin, Q., R. Fu, W. Yuan, Q. Liu, and S. Yu. 2018. "Predictive Intelligent Driver Model for Eco-Driving Using Upcoming Traffic Signal Information." Physica A: Statistical Mechanics and its Applications 508: 806-23. doi:10.1016/j.physa.2018.05.138.

Yang, K., M. Menendez, and S. I. Guler. 2018. "Implementing Transit Signal Priority in a Connected Vehicle Environment with and without Bus Stops." Transportmetrica B: Transport Dynamics 7 (1): 423-45. doi:10.10 80/21680566.2018.1434019.

Yu, H., D. Chen, Z. Wu, X. Ma, and Y. Wang. 2016. "Headway-based Bus Bunching Prediction Using Transit Smart Card Data." Transportation Research Part C: Emerging Technologies 72: 45-59.

Yu, S., R. Fu, Y. Guo, Q. Xin, and Z. Shi. 2019. "Consensus and Optimal Speed Advisory Model for Mixed Traffic at an Isolated Signalized Intersection." Physica A: Statistical Mechanics and its Applications 531. doi:10.1016/j. physa.2019.121789.

Zhang, B., G. Guo, L.-Y. Wang, and Q. Wang. 2018. "Vehicle Speed Planning and Control for Fuel Consumption Optimization with Traffic Light State." Acta Automatica Sinica 44 (3): 461-70. doi: 10.16383/j.aas.2018. c160684.

Zhao, L., S. I. Chien, L. N. Spasovic, and X. Liu. 2018. "Modeling and Optimizing Urban Bus Transit Considering Headway Variation for Cost and Service Reliability Analysis." Transportation Planning and Technology 41 (7): 706-23. doi:10.1080/03081060.2018.1504181.

\section{About the Authors}

Qi Xin (xinqi@chd.edu.cn) is an associate professor of transportation engineering at the School of Automobile, Chang'an University. He received a BS, MS, and PhD in control theory and engineering from the Northwestern Polytechnical University, Xi'an, China, in 2009, 2012, and 2016, respectively. Research interests include trajectory planning and control of autonomous and connected vehicles, and operation control of connected buses. 
Rui Fu (furui@chd.edu.cn) is a professor of transportation engineering at the School of Automobile, Chang'an University. She received a BS, MS, and PhD in vehicle application engineering from the jilin University of Technology, Changchun, China, in 1985, 1989, and 1996, respectively. Research interests include human factors, driving behavior, human-machine collaboration control, and vehicle active safety.

Shaowei Yu (swyu2016@chd.edu.cn) is a professor of transportation engineering at the College of Transportation Engineering, Chang'an University. He received a PhD in transportation information and control engineering from the Northwestern Polytechnical University, Xi'an, China, in 2016. Research interests include car-following behavior modeling and cooperative operation control of public transport.

Satish V. Ukkusuri (sukkusur@purdue.edu) is a professor at the Lyles School of Civil Engineering, Purdue University. He works in the area of transportation network modeling and disaster management. He is the director of the Purdue Interdisciplinary Transportation Modeling and Analytics Laboratory. Research interests include complex networks, intelligent transportation systems, sustainable transportation, big data analytics, disaster management, and freight transportation and logistics.

Rui Jiang (jiangrui@bjtu.edu.cn) is a professor at the School of Traffic \& Transportation, Beijing Jiaotong University. He received a BE and PhD from the University of Science and Technology of China, Hefei, China, in 1998 and 2003, respectively. He was a Humboldt fellow (2005-2006) and a Japanese Society for Promotion of Science research fellow (2008-2009). Research interests include traffic flow theory and experimental validation, intelligent transportation systems. 